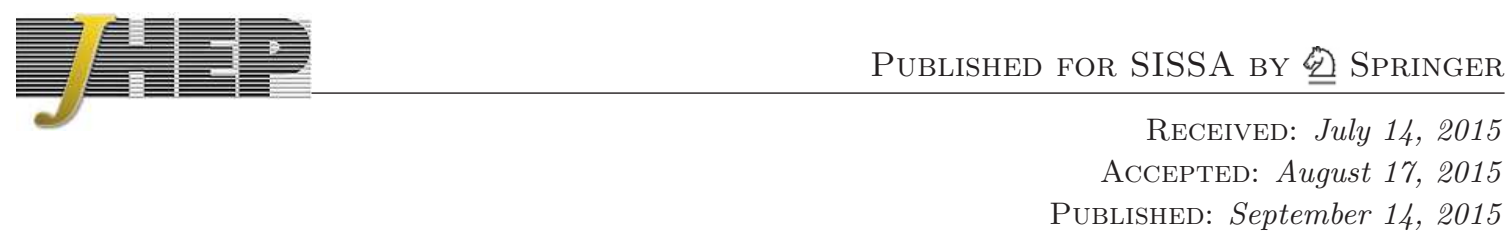

\title{
Structure of six-dimensional microstate geometries
}

\author{
Paul de Lange, Daniel R. Mayerson and Bert Vercnocke \\ Institute for Theoretical Physics, University of Amsterdam, \\ Science Park 904, Postbus 94485, 1090 GL Amsterdam, The Netherlands \\ E-mail: p.delange@uva.nl, d.r.mayerson@uva.nl, bert.vercnocke@uva.nl
}

ABSTRACT: We investigate the structure of smooth and horizonless microstate geometries in six dimensions, in the spirit of the five-dimensional analysis of Gibbons and Warner [arXiv:1305.0957]. In six dimensions, which is the natural setting for horizonless geometries with the charges of the D1-D5-P black hole, the natural black objects are strings and there are no Chern-Simons terms for the tensor gauge fields. However, we still find that the same reasoning applies: in absence of horizons, there can be no smooth stationary solutions without non-trivial topology. We use topological arguments to describe the Smarr formula in various examples: the uplift of the five-dimensional minimal supergravity microstates to six dimensions, the two-charge D1-D5 microstates, and the non-extremal JMaRT solution. We also discuss D1-D5-P superstrata and confirm that the Smarr formula gives the same result as for the D1-D5 supertubes which are topologically equivalent.

KEYwords: Black Holes in String Theory, Black Holes

ARXIV EPRINT: 1504.07987 


\section{Contents}

1 Introduction 1

2 Smarr formula in six dimensions 4

2.1 Komar integrals 4

2.2 ADM integrals 4

2.3 Normalization of the Komar integrals 5

2.4 Six-dimensional supergravity 6

2.4.1 Minimal supergravity with $n_{T}$ tensor multiplets 6

$\begin{array}{lll}2.4 .2 & \text { Smarr formula } & 7\end{array}$

2.4.3 One tensor multiplet $\quad 8$

2.4.4 Supersymmetry 8

3 Supersymmetric examples $\quad 9$

$\begin{array}{llr}3.1 & \text { General expectations } & 10\end{array}$

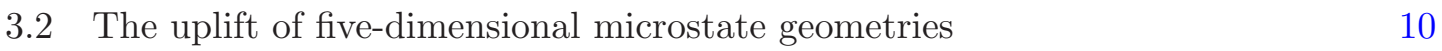

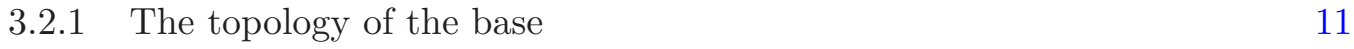

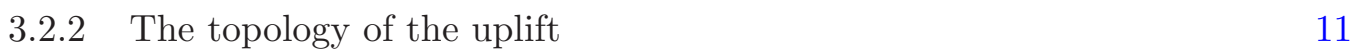

$\begin{array}{lll}3.3 & \text { D1-D5 microstate geometries and supertubes } & 13\end{array}$

$\begin{array}{lll}\text { 3.3.1 Topology and homology } & 14\end{array}$

$\begin{array}{ll}\text { 3.3.2 Cohomology and null charge } & 14\end{array}$

$\begin{array}{lll}3.4 & \text { D1-D5-P superstrata } & 15\end{array}$

4 Non-extremal example $\quad 17$

$\begin{array}{lll}4.1 & \text { Metric and gauge fields } & 17\end{array}$

$\begin{array}{lll}4.2 & \text { Constraints for smooth solutions } & 18\end{array}$

$\begin{array}{lll}4.3 & \text { Komar integral } & 19\end{array}$

5 Discussion and outlook $\quad 20$

A Uplift of five-dimensional multi-center solutions 22

A.1 General reduction $\quad 22$

A.2 Uplifting SUSY solutions 23

B Rigid $T^{4}$ reduction of IIB and $\mathrm{SO}(1,2)$ truncation $\quad 24$

\section{Introduction}

The fuzzball programme argues that extended objects of string theory alter the horizon of black holes quite drastically. ${ }^{1}$ Classical solutions to the supergravity equations of motion

\footnotetext{
${ }^{1}$ For reviews, see [1-6]. Related arguments instigated the recent firewall discussion [7-9].
} 
have played a significant role in shaping fuzzball discussions. These 'microstate geometries' are smooth, globally hyperbolic, and solitonic stationary solutions that carry the same charges as a black hole and are argued to correspond to the gravitational interpretation of certain black hole microstates.

The very existence of such smooth solitonic solutions was a bit puzzling. Based on Smarr formulas, many no-go theorems have been proven in the past for regular, stationary solutions in four dimensions, also in supergravity [10]. They can be summarized through the slogan: no solitons without horizons. However, based on explicit construction, it has become clear that there are many supersymmetric horizonless smooth geometries with charges supported by topological fluxes. Also, families of near-supersymmetric microstate geometries are supported by fluxes on non-trivial two-cycles [11, 12].

The seemingly paradoxical existence of these horizonless microstate geometries was further clarified by Gibbons and Warner in [13]. They revisited the Smarr formula in detail for five-dimensional asymptotically flat supergravity solutions and explained the mechanism that supports mass in a stationary, horizonless soliton. Besides receiving contributions from horizons, the Komar mass is also affected by previously neglected terms arising from spatial sections with non-trivial second cohomology. This is possible due to the existence of ChernSimons terms in the action and cohomologically supported fluxes. The key slogan must be modified to no solitons without horizons or topology and fluxes, which then holds regardless of supersymmetry and is in particular also valid for stationary solutions carrying the charges of a black hole with non-zero Hawking temperature. This has been further corroborated by the similar M-theory analysis of [14] and its compactification to five dimensions.

In this paper we want to discuss the topological Smarr formula in the six-dimensional arena appropriate to current microstate geometry research for the three-charge black hole. In addition, we want to explore non-extremal solutions, which have not been discussed before from the viewpoint of considering their topological contributions to the mass. ${ }^{2}$

The three charge black hole has five non-compact dimensions. It can, for instance, be obtained in IIB string theory on $T^{4} \times S^{1}$ with D1-D5-P charges on the internal directions. The string theory microstates dual to this black are given in the D1-D5 orbifold CFT. The discussion of the Smarr formula of [13] concentrated on the five-dimensional microstate geometries, which fit in five-dimensional $\mathcal{N}=1$ supergravity with three vector fields in $\mathrm{U}(1)^{3}$, after dimensional reduction on $T^{4} \times S^{1}$. However, the geometric interpretation of the D1-D5-P microstates can in principle excite the full ten-dimensional spacetime and does not have to allow a five-dimensional truncation. Indeed, the microstate geometries for the two-charge D1-D5 black hole, or 'supertubes', depend on functions of one variable, the coordinate along the $S^{1}$. These functions describe a profile in the 8 spatial components orthogonal to $S^{1}$ (four non-compact dimensions [16-18] and four torus directions [19]) and all IIB supergravity fields are excited.

A similar story is expected to hold for the microstates of the three-charge D1-D5-P black hole. As argued in [20], the generic microstate geometries in the D1-D5-P frame are expected to be described by so-called superstrata. These should be obtained from

\footnotetext{
${ }^{2}$ With the exception of the BPS-bound violating solution of [15] discussed in [13].
} 
adding momentum modes on two-charge D1-D5 supertubes and depend on functions of two variables. Their solution space might even give a leading contribution to the BekensteinHawking entropy of the black hole [21]. Arbitrary superstrata excitations are most likely computationally beyond our reach and therefore the search for solutions has been focused on keeping the $T^{4}$ rigid. The rigourous treatment of [22] shows that any solution sharing the supersymmetries of the D1-D5-P brane system in IIB then fits in six-dimensional $\mathcal{N}=(1,0)$ supergravity with two tensor multiplets, confirming earlier results of $[19,23-$ 25]. ${ }^{3}$ This may be somewhat surprising, since the D1-D5-P black hole and many of its microstate geometries only excite one tensor multiplet. Nonetheless, this extra tensor multiplet helps to smoothen singularities in supergravity solutions $[29,30]$ and was in fact a key ingredient for the first successful construction of a superstratum [31].

We extend the five-dimensional results of [13] to the framework relevant for the more abundant conjectured six-dimensional solutions. The Komar integral that gives the conserved charge for a Killing vector again has a contribution for non-trivial topology, depending on the third cohomology of space. There are several new features in our discussion:

- New asymptotics: the natural black object in our discussion is the six-dimensional D1-D5-P black string, where the string wraps the compact $S^{1}$ (compactification along $S^{1}$ gives the three-charge black hole). Hence we do not consider asymptotically flat $\mathbb{R}^{5,1}$ spacetimes, but rather focus on $\mathbb{R}^{4,1} \times S^{1}$ asymptotics. This also implies a different relation between the Komar integral and Smarr formula. For an extended object such as the black string, we cannot just relate the appropriate Komar integrals to the ADM mass, but rather to combinations of both the integrated energy density and tension of the string [32-34].

- For supersymmetric solutions: in six dimensions these always have a null Killing vector [35] but not necessarily a timelike one as in five dimensions. The Komar integral for a null Killing vector does not give the ADM mass, but rather a 'null charge'. We discuss the interpretation of this charge and its relation to the mass. As examples, we discuss the uplift of the 5D microstate geometries of [13], D1-D5 two-charge geometries, and D1-D5-P superstrata. We find that the Komar integral for the null charge is independent of the momentum charge P. This is natural as D1-D5-P superstrata and D1-D5 supertubes share the same topology: both describe "wiggles" of a topological three-sphere.

- For non-supersymmetric solutions: we explore the JMaRT solutions [36] which have a timelike Killing vector. The Komar integral gives the ADM mass. The JMaRT solitons are only smooth in six dimensions (not in five or four). As in the D1-D5 solutions, the flux through a non-contractible $S^{3}$ supports the charges.

The rest of this paper is organized as follows. In section 2 we discuss the Komar integrals and the Smarr formula. We revisit brane-like solutions with compact dimensions and their energy densities and tensions. We normalize the Komar integral in terms

\footnotetext{
${ }^{3}$ The earliest three-charge geometries were six-dimensional by construction [26-28], but lacked the intricacy of superstrata that has the potential of a solution space with large entropic contribution.
} 
of these physical quantities and highlight the difference between using timelike and null Killing vectors. Then we discuss the Komar integral in six-dimensional supergravity. We illustrate the general formula with supersymmetric examples in section 3 , and the nonsupersymmetric JMaRT solutions [36] in section 4. The supersymmetric examples include the uplift of the five-dimensional multi-center solutions discussed in [13] and the D1-D5 Lunin-Mathur geometries [16-18]; we also comment on D1-D5-P superstrata. We end with a discussion in section 5. Appendix A contains the details of the uplift to six dimensions of five-dimensional multi-center solutions and in appendix $\mathrm{B}$ we give the details of the truncation of IIB supergravity to six-dimensional supergravity with two tensor multiplets.

\section{Smarr formula in six dimensions}

We discuss Komar integrals, the relation to the energy and tension of a solution, a Smarr formula for smooth horizonless solutions using topology and their application to six-dimensional supergravity with tensor multiplets.

\section{$2.1 \quad$ Komar integrals}

Any Killing vector $K$ of a metric on a $D$-dimensional Lorentzian spacetime defines a conserved quantity through a Komar integral:

$$
\mathcal{Q}_{K}=\frac{1}{8 \pi G_{D}} \int_{\partial V_{\infty}} \star d K=\frac{1}{8 \pi G_{D}} \int_{\partial V_{\infty}}\left(\partial_{\mu} K_{\nu}-\partial_{\mu} K_{\nu}\right) d \Sigma^{\mu \nu}
$$

where we integrate over a closed spatial surface at infinity. Killing vectors enjoy the property $\nabla^{2} K_{\mu}=-R_{\mu \nu} K^{\nu}$. With the help of Stokes' theorem, we can then rewrite this as a bulk integral over a volume $V$ on a spatial hypersurface with boundary $\partial V_{\infty} \cup \partial V_{\text {int }}$ :

$$
\mathcal{Q}_{K}=-\frac{1}{4 \pi G_{D}} \int_{V} \star\left(K^{\mu} R_{\mu \nu} d x^{\nu}\right)-\frac{1}{8 \pi G_{D}} \int_{\partial V_{\text {int }}} d S^{\mu \nu}\left(\partial_{\mu} K_{\nu}-\partial_{\mu} K_{\nu}\right) .
$$

For a spacetime with a timelike Killing vector $K$, one usually relates the Komar integral to the ADM mass. However, this is only valid for an energy-momentum tensor that asymptotically approaches that of a weak static dust source, with $T_{00} \gg T_{0 i}, T_{i j}$ and $\partial_{0} g_{i j}=0$ asymptotically. For a string-like object spanning the $y$ direction, we expect that $T_{00}$ and $T_{y y}$ will be of the same order, so we need to slightly modify the story.

\subsection{ADM integrals}

We now review the relevant results of [34]. To relate the Komar integral to physical quantities such as the ADM energy, we consider an energy-momentum tensor that has asymptotically $p+1$ dominating diagonal components $T_{00}, T_{a a}, a=1 \ldots p$ and $p<D-3$. We assume all other components of the energy-momentum tensor are subleading compared to these. We take the $p$ coordinates to be compact and consider the linearization around a flat metric, $g_{\mu \nu}=\eta_{\mu \nu}+h_{\mu \nu}$ with Minkowski reference metric

$$
d s_{D}^{2}=-d t^{2}+\sum_{a=1}^{p} d y^{a} d y^{a}+\sum_{i=1}^{n} d x^{i} d x^{i}, \quad n=D-p-1 .
$$


We write the Einstein equations as

$$
R_{\mu \nu}-\frac{1}{2} R g_{\mu \nu}=8 \pi G_{D} T_{\mu \nu}
$$

The energy density $\mathcal{E}$, average tension $\mathcal{T}$ and angular momentum density $\mathcal{J}$ are

$$
\begin{aligned}
\mathcal{E} & =\int d^{n} x\left\langle T_{00}\right\rangle, \\
\mathcal{T} & =-\frac{1}{p} \sum_{a=1}^{p} \int d^{n} x\left\langle T_{a a}\right\rangle, \\
\mathcal{J}_{i j} & =\int d^{n} x\left(x_{i}\left\langle T_{j 0}\right\rangle-x_{j}\left\langle T_{i 0}\right\rangle\right) .
\end{aligned}
$$

with the average over the compact space $\langle X\rangle=1 / V_{p} \int d^{p} y X$. From the linearized Einstein equations, one can then deduce the relations to the linearized metric components $h_{\mu \nu}$ [34]:

$$
\begin{aligned}
& \mathcal{E}=-\frac{1}{16 \pi G_{D}(n-2)} \int_{\partial V_{\infty}} d S_{i} \partial_{i}\left((n-1) h_{00}-h_{a a}\right), \\
& \mathcal{T}=-\frac{1}{p} \frac{1}{16 \pi G_{D}(n-2)} \int_{\partial V_{\infty}} d S_{i} \partial_{i}\left(p h_{00}-(n+p-2) h_{a a}\right) .
\end{aligned}
$$

These are the formulae that relate the asymptotic expansion of an extended object (where $T_{a a}$ is not negligible compared to $\left.T_{00}\right)$ to its mass and tension. After dimensional reduction over the $p$ internal directions, the ADM mass in $D-p$ dimensions is given by $\mathcal{E}$. The angular momentum density can still be read off from the off-diagonal metric components:

$$
g_{0 i}=\frac{16 \pi G_{D}}{\Omega_{D-2}} \frac{x^{j} J^{j i}}{\rho^{n}}+\ldots
$$

where $\Omega_{D-2}$ is the volume of the unit $(D-2)$-sphere and $\rho$ the radius in the four spatial dimensions.

\subsection{Normalization of the Komar integrals}

We now discuss the relation of the Komar integral to the energy density and tension.

Timelike Killing vector. One readily shows that for a timelike Killing vector $K$ that asymptotes to $K_{\infty}=\partial_{t}$, we have the normalization

$$
\mathcal{E}-\frac{p}{(D-3)} \mathcal{T}=-\frac{1}{16 \pi G_{D}} \frac{(D-2)}{(D-3)} \int_{\partial V_{\infty}} d S_{\mu \nu}\left(\partial^{\mu} K^{\nu}-\partial^{\nu} K^{\mu}\right) .
$$

For $p=0$, we retrieve the usual relations between the ADM mass $M=\mathcal{E}$ and the asymptotic form of the metric components $[13,37]$

$$
\begin{aligned}
g_{00} & =-1+\frac{16 \pi G_{D}}{(D-2) \Omega_{D-2}} \frac{M}{\rho^{D-3}}+\ldots \\
g_{i j} & =\left(1+\frac{16 \pi G_{D}}{(D-2)(D-3) \Omega_{D-2}} \frac{M}{\rho^{D-3}}\right) \delta_{i j}+\ldots
\end{aligned}
$$


Null Killing vector. Most of this paper is concerned with supersymmetric solutions in six dimensions. For these, it is useful to discuss $p=1$ and consider null coordinates:

$$
u=\frac{t-y}{\sqrt{2}}, \quad v=\frac{t+y}{\sqrt{2}} .
$$

For a null Killing vector $K$ that asymptotically becomes $K_{\infty}=\partial_{u}$, one finds:

$$
\mathcal{E}+\mathcal{T}=-\frac{1}{8 \pi G_{D}} \frac{(n+p-1)}{(n-2)} \int_{\partial V_{\infty}} d S_{\mu \nu}\left(\partial^{\mu} K^{\nu}-\partial^{\nu} K^{\mu}\right)
$$

Note that these results, as in [34], are in principle only valid for time-independent metric perturbations. Metrics with a null Killing vector $\partial_{u}$ do not in general have to be timeindependent. However, the time-dependence of the metric is heavily constrained. Since we average (integrate) over the internal, compact direction $y$, the resulting averaged metric must be time-independent and the results for the Komar integrals remain valid.

The normalization of the Komar integral (2.1), which we use in a $6 \mathrm{D}$ supergravity context for strings $(p=1)$, implies that:

$$
\mathcal{Q}_{K}=-\frac{1}{2}(\mathcal{E}+\mathcal{T})
$$

\subsection{Six-dimensional supergravity}

Here we discuss the six-dimensional setup relevant for the three-charge black hole. First we consider an arbitrary number $n_{T}$ of tensor multiplets; for superstrata in six dimensions, $n_{T}=2$. We also explicitly give the formulas for $n_{T}=1$, which is relevant for all of the examples we discuss except the superstrata of section 3.4.

\subsubsection{Minimal supergravity with $n_{T}$ tensor multiplets}

The six-dimensional supergravity theories of relevance to this work have an $\mathrm{SO}(n, m)$ global symmetry, with $n$ the number of tensors in the gravity multiplet. In the D1-D5-P frame, the relevant six-dimensional theories are obtained by a compactification on $T^{4}$ or K3, which respectively give $\mathcal{N}=(2,2)$-supergravity with $\mathrm{SO}(5,5)$ global symmetry and $\mathcal{N}=(2,0)$ supergravity with an $\mathrm{SO}(5,21)$ symmetry group.

Luckily, we do not need the full details of these extended supergravity theories. Rather, we can consider a consistent truncation to 'minimal' six-dimensional supergravity with only $\mathcal{N}=(1,0)$ supersymmetry. This theory has $\mathrm{SO}\left(1, n_{T}\right)$ global symmetry where $n_{T}$ is the number of tensor multiplets and is in principle arbitrary as it is unfixed by supersymmetry. For our purposes, $n_{T}$ will be either 1 or 2 , see appendix B for more details on the reduction from $10 \mathrm{D}$. Even though we focus on the theory with $\mathrm{SO}\left(1, n_{T}\right)$ global symmetry, our results and in particular the Komar integrals (2.24) and (2.25) below are straightforwardly extended to the bosonic sector of six-dimensional supergravity theories with more supersymmetry, by formally replacing the $\mathrm{SO}\left(1, n_{T}\right)$ metric $\eta_{r s}$ with the metric of the appropriate global symmetry group. 
When $n_{T}>1$, the equations of motion of the tensor fields do not follow from an action. We can still consider the 'pseudo-action' $[38,39]$ for the bosonic fields ${ }^{4}$

$$
\mathcal{L}=\frac{1}{4} R-\frac{1}{2} \partial_{\mu} v_{r} \partial^{\mu} v^{s}-\frac{1}{12} \mathcal{M}_{r s} G_{\mu \nu \rho}^{r} G^{s \mu \nu \rho},
$$

that captures the equations of motion of the scalar fields and the metric. The scalars parametrize the coset $\mathrm{SO}\left(1, n_{T}\right) / \mathrm{SO}\left(n_{T}\right)$. They can be organized in the $\mathrm{SO}\left(1, n_{T}\right)$-matrix $V=\left(\begin{array}{c}v_{r} \\ x_{r}^{M}\end{array}\right)$ with $M=1 \ldots n_{T}$ and $r=0 \ldots n_{T} .{ }^{5}$ They enter the tensor dynamics through the scalar metric $\mathcal{M}=\eta V^{T} V \eta$, with $\eta$ the $\mathrm{SO}\left(1, n_{T}\right)$-metric, or in index notation

$$
\mathcal{M}_{r s}=v_{r} v_{s}+x_{r}^{M} x_{s}^{M} .
$$

The dynamics of the $n_{T}+1$ tensor fields $G^{r}$ are captured by the self-duality conditions and Bianchi identities

$$
\mathcal{M}_{r s} G^{s}=\eta_{r s} \star G^{s}, \quad d G^{r}=0,
$$

where $\star$ is the six-dimensional Hodge star operator. Finally, the Einstein equations are:

$$
R_{\mu \nu}=2 \partial_{\mu} v^{r} \partial_{\nu} v_{r}+\frac{1}{2} \mathcal{M}_{r s} G_{\mu \alpha \beta}^{r} G_{\nu}^{s \alpha \beta} .
$$

\subsubsection{Smarr formula}

We are concerned with field configurations that respect the symmetry of a Killing vector $K$. This means the Lie derivative of the fields with respect to $K$ vanishes:

$$
\mathcal{L}_{K} g_{\mu \nu}=0, \quad \mathcal{L}_{K} v^{r}=0, \quad \mathcal{L}_{K} G^{r}=0 .
$$

Since $d G^{r}$ and $\mathcal{L}_{K}=d i_{K}+i_{K} d$, we can write the three-form and its dual as

$$
i_{K} G^{r}=d \Lambda^{r}+H^{r}
$$

for some globally defined one-forms $\Lambda^{r}$ and closed but not exact two-forms $H^{r}$. The Einstein equations (2.20) become

$$
K^{\mu} R_{\mu \nu}=\frac{1}{2} \nabla_{\rho}\left(\mathcal{M}_{r s} \Lambda_{\sigma}^{r} G_{\nu}^{s \rho \sigma}\right)+\frac{1}{2}\left(\mathcal{M}_{r s} H_{\rho \sigma} G_{\nu}^{s \rho \sigma}\right) .
$$

Then the Komar integral (2.2) is:

$$
\mathcal{Q}_{K}=-\frac{1}{8 \pi G_{6}} \int_{V} \mathcal{M}_{r s} H_{\rho \sigma}^{r} G_{\nu}^{s \rho \sigma} d V^{\nu}-\frac{1}{8 \pi G_{6}} \int_{\partial V_{\mathrm{int}}}\left(\mathcal{M}_{r s} \Lambda_{\sigma}^{r} G_{\mu \nu}^{s \sigma} d S^{\mu \nu}+\left(\partial_{\mu} K_{\nu}-\partial_{\mu} K_{\nu}\right)\right) .
$$

As in [13], we find that we can support matter (non-zero Komar integrals) with horizons or with topology. For trivial topology, $H^{r}=0$ and the Smarr formula (2.24) relates the Komar integral to horizon quantities (area, charges and angular momenta). If also no

\footnotetext{
${ }^{4}$ To avoid confusion with standard notation $H$ for harmonic forms, we do not follow the notation of [38, 39] for the three-forms and the kinetic matrix. To convert, use $G^{r}=H_{\text {theirs }}^{r}$ and $\mathcal{M}_{r s}=\left(G_{r s}\right)_{\text {theirs }}$.

${ }^{5}$ It is customary to write the $\mathrm{SO}\left(1, n_{T}\right)$ conditions $V \eta V^{T}=V^{T} \eta V=\eta$ in component notation as $v_{r} v^{r}=1, v^{r} x_{r}^{M}=0, v_{r} v_{s}-x_{r}^{M} x_{s}^{M}=\eta_{r s}$.
} 
horizons are present, the right-hand side of (2.24) is zero and we get a vanishing Komar integral for the Killing vector $K$.

We are interested in spacetimes without inner boundaries. With (2.19), we find

$$
\mathcal{Q}_{K}=-\frac{1}{4 \pi G_{6}} \int_{V} \eta_{r s} H^{r} \wedge G^{s},
$$

so that only non-trivial topology can allow for non-zero Komar integrals.

\subsubsection{One tensor multiplet}

For many of the solutions in this paper we can restrict to $\mathrm{SO}(1,1)$ supergravity with $n_{T}=1$. Including only one extra tensor multiplet in addition to the minimal supergravity multiplet is convenient as it allows for a Lagrangian description of the theory. The single self-dual three-form $G^{+}$of the gravity multiplet can be combined with the single anti self-dual threeform $G^{-}$of the tensor multiplet in one unconstrained three-form $G=\frac{1}{2}\left(G^{+}+G^{-}\right)$. The action becomes

$$
\mathcal{L}=\frac{1}{4} R-\frac{1}{2} \partial_{\mu} X \partial^{\mu} X-\frac{1}{12} e^{2 \sqrt{2} X} G_{\mu \nu \rho} G^{\mu \nu \rho} .
$$

We introduce the dual three-form (equivalent to (2.19)):

$$
\tilde{G}=e^{2 \sqrt{2} X} \star G .
$$

To compare to the discussion of section 2.4.1, we can choose $G^{0}=G, G^{1}=\tilde{G}$. The $\operatorname{SO}(1,1)$ metric is then $\eta=\sigma_{1}$, and one can choose the $\mathrm{SO}(1,1)$ scalar matrix as $V=\exp \left(\sqrt{2} X \sigma_{3}\right)$, where $\sigma_{i}$ are the Pauli matrices.

The Einstein equation can be (re)written as:

$$
R_{\mu \nu}=2 \partial_{\mu} X \partial_{\nu} X+\frac{1}{2}\left(e^{2 \sqrt{2} X} G_{\mu a b} G_{\nu}^{a b}+e^{-2 \sqrt{2} X} \tilde{G}_{\mu a b} \tilde{G}_{\nu}^{a b}\right) .
$$

The Komar integral (2.25) is then

$$
\mathcal{Q}_{K}=-\frac{1}{4 \pi G_{6}} \int_{V}(H \wedge \tilde{G}+\tilde{H} \wedge G),
$$

with the harmonic forms $H, \tilde{H}$ defined through

$$
i_{K} G=d \Lambda+H, \quad \tilde{i}_{K} \tilde{G}=d \tilde{\Lambda}+\tilde{H}
$$

for some global one-forms $\Lambda$.

\subsubsection{Supersymmetry}

Let us also mention the fermionic content of the $\mathrm{SO}(1,1)$ theory. The gravity multiplet consists of $\left(e_{\mu}, \psi_{\mu}^{\alpha}, B_{\mu \nu}^{+}\right)$with $B^{+}$a self-dual tensor such that $G^{+} \equiv d B^{+}=\star G^{+}$. The tensor multiplet consists of $\left(B_{\mu \nu}^{-}, \chi^{\alpha}, X\right)$ with $G^{-} \equiv d B^{-}=-\star G^{-}$. The supersymmetry transformations of the fermions are:

$$
\begin{aligned}
\delta \psi_{\mu}^{\alpha} & =\left(\partial_{\mu}-\frac{1}{4} e^{\sqrt{2} X} G_{\mu \nu \sigma}^{+} \gamma^{\nu \sigma}\right) \varepsilon^{\alpha} \\
\delta \chi^{\alpha} & =\frac{1}{2 i}\left(\sqrt{2} \gamma^{\mu} \partial_{\mu} X+\frac{1}{6} e^{\sqrt{2} X} G_{\mu \nu \rho}^{-} \gamma^{\mu \nu \rho}\right) \varepsilon^{\alpha} .
\end{aligned}
$$


Given a Killing spinor $\epsilon^{\alpha}$ we can construct the bilinear vector:

$$
K_{\mu} \varepsilon^{\alpha \beta}=\bar{\epsilon}^{\alpha} \gamma_{\mu} \varepsilon^{\beta},
$$

which is always a null Killing vector, $K \cdot K=0$. The supersymmetry equations imply (using the form notation $K \equiv K^{\mu} g_{\mu \nu} d x^{\nu}$ ):

$$
\begin{aligned}
d K & =2 e^{\sqrt{2} X} i_{K} G^{+}=i_{K}\left(e^{\sqrt{2} X} G+e^{-\sqrt{2} X} \tilde{G}\right), \\
i_{K} d X & =0,
\end{aligned}
$$

since the self-dual part of $G$ is given by $G^{+}=1 / 2\left(G+e^{-2 \sqrt{2} X} \tilde{G}\right)$. Using $i_{K} \star G=\star(G \wedge K)$, this allows us to write the null charge associated with $K$ as

$$
\mathcal{Q}_{K}=\frac{1}{8 \pi G_{6}} \int_{\partial V_{\infty}} \star d K=-\frac{1}{8 \pi G_{6}} \int_{\partial V_{\infty}}(\tilde{G}+G) \wedge K
$$

where we have assumed that $X=0$ at infinity, which we can always do for asymptotically flat spacetimes. In the microstate geometries of section 3, we find that $\partial V_{\infty}=S^{1} \times S^{3}$, and the Killing vector $K$ projected on this spacelike surface is (proportional to) the isometry along the compact $S^{1}$. In the notation of the metric (3.1) below, $K=-d v$ at spatial infinity. This means we simply get:

$$
\mathcal{Q}_{K}=-\frac{L_{v}}{8 \pi G_{6}} \int_{S^{3}}(\tilde{G}+G)=-\frac{L_{v} \pi}{4 G_{6}}\left(Q_{e}+Q_{m}\right),
$$

where $L_{v}=2 \pi R_{v}$ is the size of the $S^{1}$ direction parametrized by $v$ (at constant time). This relation is thus simply the BPS condition in $6 \mathrm{D}$ relating the null charge associated to $K$ to the electric and magnetic charges of the solution.

\section{Supersymmetric examples}

We now analyze in detail the null Komar integral for known smooth supersymmetric solutions to six-dimensional supergravity. The structure of supersymmetric solutions in $6 \mathrm{D}$ minimal supergravity was studied in [35] and including an additional vector multiplet and one tensor multiplet in [40]. Using the Killing spinors of such supersymmetric solutions, one can always construct a null Killing vector which locally is $V=\partial_{u}$. The metric can then be shown to take the form:

$$
d s_{6}^{2}=-2 H^{-1}\left(d v+\beta_{i} d x^{i}\right)\left[d u+\omega_{i} d x^{i}+\frac{\mathcal{F}}{2}\left(d v+\beta_{i} d x^{i}\right)\right]+H d x_{4}^{2},
$$

where $d x_{4}$ is the line element on the $4 \mathrm{D}$ "base space" $\mathcal{B}$, the one-forms $\beta=\beta_{i} d x^{i}, \omega=\omega_{i} d x^{i}$ only have legs on $\mathcal{B}$ and the functions $H, \beta_{i}, \omega_{i}, \mathcal{F}$ are in general functions of $v$ and all of the $4 \mathrm{D}$ base coordinates $x^{i}$. The conditions that these functions (and the three-form and scalar) must satisfy for supersymmetric solutions can be found in [40], or [41] whose conventions and notation we follow. Note that the ansatz (3.1) only holds for sections 3.1-3.3, in section 3.4 we extend the ansatz for two tensor multiplets. 


\subsection{General expectations}

It is instructive to first work out the ADM integrals $\mathcal{E}$ and $\mathcal{T}$ for the three-charge solutions of our interest. Asymptotically, the metric (3.1) approaches that of the three-charge black string for which $H=\left(Z_{2} Z_{3}\right)^{-1 / 2}, \mathcal{F}=-Z_{1}, \omega=0, \beta=0$ and $Z_{i}=1+Q_{i} / \rho^{2}$, with $\rho$ the standard radial coordinate of the $4 \mathrm{D}$ base $\mathcal{B}=\mathbb{R}^{4}$. The asymptotic metric perturbation in the coordinates $t, y(2.14)$ is

$$
h_{00}=\frac{1}{2} \frac{Q_{2}+Q_{3}+Q_{1}}{\rho^{2}}+O\left(\rho^{3}\right), h_{y y}=\frac{1}{2} \frac{-Q_{2}-Q_{3}+Q_{1}}{\rho^{2}}+O\left(\rho^{3}\right) .
$$

and we find that

$$
\mathcal{E}=\frac{\pi L_{y}}{4 \pi G_{6}}\left(Q_{2}+Q_{3}+\frac{1}{2} Q_{1}\right), \quad \mathcal{T}=\frac{\pi L_{y}}{4 \pi G_{6}}\left(Q_{2}+Q_{3}-\frac{1}{2} Q_{1}\right),
$$

with $y \sim y+L_{y}$. Note that $\mathcal{E}$ is the ADM mass after dimensional reduction over the $y$-circle. ${ }^{6}$ Using (2.16), we anticipate that the Komar integral will be:

$$
\mathcal{Q}_{K}=-\frac{1}{2}(\mathcal{E}+\mathcal{T})=-\frac{\pi L_{y}}{4 G_{6}}\left(Q_{2}+Q_{3}\right),
$$

and does not involve the momentum charge $Q_{1}$.

\subsection{The uplift of five-dimensional microstate geometries}

As a warm-up, we consider the uplift of five-dimensional microstate geometries. Komar integrals and Smarr formulae for those geometries were discussed at length in [13], hence we do not go into much detail here. The solutions are completely smooth multi-centered solutions of the 5D STU model with three gauge fields $A^{I}(I=\{1,2,3\})$ and three scalars $X^{I}$, constrained by $X^{1} X^{2} X^{3}=1$. The $5 \mathrm{D}$ Lagrangian is given by (A.11). The $6 \mathrm{D}$ theory of minimal supergravity coupled to one tensor multiplet (2.26) gives exactly this STU model when dimensionally reduced to 5D. See appendix A for more details.

The $5 \mathrm{D}$ solutions that we are interested in are given by the metric [42-44]:

$$
d s_{5}^{2}=-Z^{-2}(d t+k)^{2}+Z d s_{4}^{2}, \quad Z=\left(Z_{1} Z_{2} Z_{3}\right)^{1 / 3} .
$$

where the $4 \mathrm{D}$ base space $\mathcal{B}$ is Gibbons-Hawking: it is a $\mathrm{U}(1)$ fibration with coordinate $\psi$ over flat $\mathbb{R}^{3}$. The solutions are then determined by specifying the poles of eight functions $V, K^{I}, L_{I}, M$, which are harmonic functions on $\mathbb{R}^{3}$. For instance, we have $Z_{I}=L_{I}+C_{I J K} K^{J} K^{K} / 2 V$ with $C_{I J K}=\left|\epsilon_{I J K}\right|$. These eight harmonic functions must satisfy stringent conditions in order for the full $5 \mathrm{D}$ spacetime to be completely regular and asymptotically flat $[1,13]$.

The gauge potentials in $5 \mathrm{D}$ are:

$$
A^{I}=-Z_{I}^{-1}(d t+k)+B^{I}
$$

\footnotetext{
${ }^{6}$ Note that the dimensional reduction in section 3.2 and appendix A.2 is a reduction over the spacelike $v$-circle, which will give a different resulting 5D ADM mass in terms of $Q_{1}$, see eq. (3.10).
} 
where $B^{I}$ is a magnetic potential (only well-defined locally). The scalars are given by:

$$
X^{I}=\frac{Z}{Z_{I}}
$$

For asymptotically flat 5D spacetimes, we have asymptotically:

$$
Z_{I} \sim 1+\frac{Q_{I}}{4 r}=1+\frac{Q_{I}}{\rho^{2}}
$$

with $r$ the usual radial coordinate on $\mathbb{R}^{3}$ and $\rho=4 r$ is the radial coordinate on the four-dimensional base. In microstate geometry literature, the charges $Q_{I}$ are normalized through the asymptotic expansion of the electric field in $5 \mathrm{D}$ as $F_{0 \rho} \sim 2 \frac{Q_{I}}{\rho^{3}}$ and not with factors involving the volume of the three sphere that are more common from Gaussian integrals. This means that we have:

$$
-\frac{1}{16 \pi G_{5}} \int_{\partial V_{\infty}} \star_{5} F_{I}=\frac{\pi}{4 G_{5}} Q_{I} .
$$

For the six-dimensional metric, scalar and tensor solutions see eqs. (A.20).

\subsubsection{The topology of the base}

The poles of $V$ ('centers') indicate where the $\psi$-fibre degenerates in the $4 \mathrm{D}$ base space (although the complete 5D spacetime is always completely smooth). Since the $\psi$-fibre degenerates at each center, we can construct non-contractible compact two-cycles in the $4 \mathrm{D}$ space, which are also compact two-cycles in the full 5D geometry. These two-cycles are constructed by taking the $\psi$-fibration over an arbitrary path in $\mathbb{R}^{3}$ between two centers. This completely determines the 5D homology structure of simply connected solutions. For $N=2 p+1$ centers, the global topology is that of a $p$-fold connected sum of $\left(S^{2} \times S^{2}\right)$ with a point removed, for $N=2 p$ centers the topology is $\left(\mathbb{R}^{2} \times S^{2}\right) \#\left(S^{2} \times S^{2}\right) \# \ldots \#\left(S^{2} \times S^{2}\right){ }^{7}$

The five-dimensional ADM mass of these solutions can be written as [13]

$$
M_{\mathrm{ADM}, 5 \mathrm{D}}=-\frac{1}{32 \pi G_{5}} C_{I J K} \alpha^{I} \int_{\Sigma_{4}} F^{J} \wedge F^{K}=\frac{\pi}{4 G_{5}} \alpha^{I} Q_{I}=\frac{\pi}{4 G_{5}}\left(Q_{1}+Q_{2}+Q_{3}\right),
$$

where $\alpha^{I}=1$ for asymptotically flat solutions and $\Sigma_{4}$ is a spacelike surface of constant time. The integral of $F^{J} \wedge F^{K}$ is computed "entirely with cohomology", by calculating the flux of the $F^{I}$ over the non-trivial compact two-cycles of the geometry as well as the intersection number of these two-cycles.

\subsubsection{The topology of the uplift}

The six-dimensional uplift of (3.5) is a non-trivial fibration of the new coordinate $v$. From the expression for the three-form:

$$
2 G=\left(X^{3}\right)^{-2} \star_{5} F^{3}+F^{2} \wedge\left(d v+A^{1}\right),
$$

\footnotetext{
${ }^{7}$ We only discuss $V=\sum_{i} q_{i} /\left|x-x_{i}\right|$ with $\left|q_{i}\right|=1$, such that the centers are smooth points in the full space, and $\sum_{i} q_{i}=1$, such that the space is asymptotically flat.
} 
we can easily see that we have:

$$
2 i_{K} G=d\left(\lambda_{2}\left(d v+A^{1}\right)\right)+d\left(Z_{1}^{-1} Z_{2}^{-1}(d t+k)\right)+F^{1},
$$

where we have defined $\lambda_{I}=Z_{I}^{-1}-1$. The form given in the first term, $\lambda_{2}\left(d v+A^{1}\right)$, is well-defined. The second term is $Z_{1}^{-1} Z_{2}^{-1}(d t+k)$ and is also a well-defined form (as discussed in [13]). This implies the cohomology split:

$$
\begin{aligned}
& 2 \Lambda=\lambda_{2}\left(d v+A^{1}\right)+Z_{1}^{-1} Z_{2}^{-1}(d t+k), \\
& 2 H=F^{1} .
\end{aligned}
$$

Similarly, we can find $\tilde{\Lambda}, \tilde{H}$ by switching the roles of $Z_{2}$ and $Z_{3}$ in the above expressions. Note that also $2 \tilde{H}=F^{1}$.

The null charge is then:

$$
\begin{aligned}
\mathcal{Q}_{K} & =-\frac{1}{4 \pi G_{6}} \int_{V}(H \wedge \tilde{G}+\tilde{H} \wedge G) \\
& =-\frac{1}{16 \pi G_{6}} \int_{V}\left(F^{1} \wedge\left(F^{3} \wedge d v\right)+F^{1} \wedge\left(F^{2} \wedge d v\right)\right) \\
& =\frac{L_{v}}{16 \pi G_{6}} \int_{\Sigma_{4}}\left(F^{1} \wedge F^{3}+F^{1} \wedge F^{2}\right) \\
& =-\frac{L_{v} \pi}{4 G_{6}}\left(Q_{2}+Q_{3}\right),
\end{aligned}
$$

where we used the cohomological computation of the integral $F^{I} \wedge F^{J}$ in 5D over $\Sigma_{4}$ [13], and $V=S^{1}(v) \times \Sigma_{4}$. We see that the null charge is simply the sum of electric and magnetic (string) charges. Note that in five dimensions, $Q_{1}$ is on the same footing as $Q_{2,3}$, but in six dimensions it is a momentum charge and does not appear in the null charge $\mathcal{Q}_{K}$.

The analysis above shows us that we clearly still have non-trivial compact two-cycles in six dimensions which are given by the trivial uplift of the two-cycles of the five-dimensional solution. These are the cycles supporting the cohomological flux $H, \tilde{H} \sim F^{1}$. The $S^{1}$ fibration of the coordinate $v$ over the compact two-cycles of the five-dimensional geometry also introduces new non-trivial three-cycles. Over these cycles, the cohomology elements $F^{2,3} \wedge d v$ have non-zero flux.

However, this is not quite the end of the story. In $6 \mathrm{D}$, we must also have a non-trivial three-sphere at infinity. Indeed, the (electric string) charge in 6D is defined as:

$$
Q_{e}=\frac{1}{2 \pi^{2}} \int_{S^{3}(\infty)} e^{2 \sqrt{2} X} \star G,
$$

where $S^{3}$ is the $S^{3}$ at infinity perpendicular to the string which is along $v$. Since the equation of motion for the three-form is simply $d\left(e^{2 \sqrt{2} X} \star G\right)=0$, this $S^{3}$ at infinity must be non-contractible to be able to support non-zero flux for smooth solutions free of singularities. Note that this non-trivial three-cycle is absent in the original 5D geometry. This can be explained by the fact that this three-cycle must be homologically equivalent to an $S^{1}(v)$ fibration over a two-cycle in the $4 \mathrm{D}$ base (which we mentioned above). These new (compared to 5D) non-trivial three-cycles in constant time-slices of the six-dimensional geometry are an interesting feature of the $S^{1}(v)$ uplift. 


\subsection{D1-D5 microstate geometries and supertubes}

We are now ready to discuss the topology and the Komar integral for more generic solutions of the D1-D5-P system. In this section, we first focus on the D1-D5 supertube solutions of Lunin and Mathur [16-18]. As we explain in section 3.4, the result (3.39) for the Komar integral is the same for more generic D1-D5 supertubes and D1-D5-P superstrata, since those describe wiggles of the D1-D5 supertube and are topologically equivalent.

The D1-D5 Lunin-Mathur geometries are solutions to six-dimensional supergravity with only one tensor multiplet:

$$
\begin{aligned}
d s^{2} & =-\frac{2}{\sqrt{Z_{1} Z_{2}}}(d v+\beta)(d u+\omega)+\sqrt{Z_{1} Z_{2}} d s_{4}^{2}, \\
e^{2 \sqrt{2} X} & =\frac{Z_{1}}{Z_{2}}, \\
2 B & =-Z_{1}^{-1}(d u+\omega) \wedge(d v+\beta)+\gamma_{2} .
\end{aligned}
$$

Here $d s_{4}^{2}$ is the $4 \mathrm{D}$ flat metric with coordinates $x_{i}(i=\{1, \ldots, 4\})$ and $a_{1}, \gamma_{2}, \beta, \omega$ are forms on the 4-manifold. The D1-D5 microstate is completely determined by profile functions $g_{i}(v), i=1 \ldots 4$ with $0 \leq v \leq L$. Certain important functions are given by (for the complete list of fields, see for example [22]):

$$
\begin{aligned}
Z_{2} & =1+\frac{Q_{5}}{L} \int_{0}^{L} \frac{1}{\left|x_{i}-g_{i}\left(v^{\prime}\right)\right|^{2}} d v^{\prime}, & Z_{1} & =1+\frac{Q_{5}}{L} \int_{0}^{L} \frac{\left|\dot{g}_{i}\left(v^{\prime}\right)\right|^{2}}{\left|x_{i}-g_{i}\left(v^{\prime}\right)\right|^{2}} d v^{\prime}, \\
A & =-\frac{Q_{5}}{L} \int_{0}^{L} \frac{\dot{g}_{j}\left(v^{\prime}\right) d x^{j}}{\left|x_{i}-g_{i}\left(v^{\prime}\right)\right|^{2}} d v^{\prime}, & d B & =-\star_{4} d A, \\
\beta & =\frac{-A+B}{\sqrt{2}}, & \omega & =\frac{-A-B}{\sqrt{2}}, \\
d \gamma_{2} & =\star_{4} d Z_{2} . & &
\end{aligned}
$$

Perhaps the easiest explicit profile is the once-wound circle, given by (with $L=2 \pi R_{y}$ ):

$$
g_{1}(v)=a \cos \left(v / R_{y}\right), \quad g_{2}(v)=a \sin \left(v / R_{y}\right), \quad g_{3}(v)=g_{4}(v)=0 .
$$

Then we can parametrize the (flat) 4D metric as:

$$
d s_{4}^{2}=\frac{f}{r^{2}+a^{2}} d r^{2}+f d \theta^{2}+\left(r^{2}+a^{2}\right) \sin ^{2} \theta d \phi^{2}+r^{2} \cos ^{2} \theta d \psi^{2},
$$

and the above functions become:

$$
\begin{array}{rlrl}
Z_{1} & =1+\frac{Q_{1}}{f}, & Z_{2} & =1+\frac{Q_{5}}{f}, \\
A & =-a \sqrt{Q_{1} Q_{5}} \frac{\sin ^{2} \theta}{f} d \phi, & B & =-a \sqrt{Q_{1} Q_{5}} \frac{\cos ^{2} \theta}{f} d \psi, \\
f & =r^{2}+a^{2} \cos ^{2} \theta, &
\end{array}
$$

where $Q_{1}=a^{2} R_{y}^{2} / Q_{5}$, and the D1-D5 string at $x_{i}=F_{i}(v)$ is now at $r=0, \theta=\pi / 2(f=0)$. 


\subsubsection{Topology and homology}

The topology of the D1-D5 system with once-wound circular profile was discussed in [18]. Any D1-D5 geometry with profile $g_{i}^{\prime}(v)$ that can be continuously deformed into a circle will share the same topology of $\mathbb{R}^{2} \times S^{3}$. At infinity we have an $S^{3}(\theta, \phi, \psi)$ of the $4 \mathrm{D}$ base, which deforms continuously to the non-trivial $S^{3}(\theta, \tilde{\phi}, \tilde{\psi})$ in the interior with $\tilde{\phi}=\phi+t / R, \tilde{\psi}=$ $\psi+y / R$, while $S^{1}(y)$ (keeping $\tilde{\psi}$ fixed) shrinks to zero size in the interior.

Hence we clearly have exactly one non-trivial three-cycle given by the three-sphere at infinity, and one non-trivial (non-compact) two cycle, given by the volume element of the $\mathbb{R}^{2}$ factor. The three-cycle is again needed in this singularity-free geometry in order for the geometry to be able to support non-zero three-form flux. The intersection number between the two-cycle and the three-cycle is simply +1 (with suitable orientations of the cycles).

\subsubsection{Cohomology and null charge}

For a general D1-D5 geometry, we have:

$$
\begin{aligned}
2 i_{k} G & =d\left(Z_{1}^{-1}(d v+\beta)\right) \\
& =\frac{1}{\sqrt{2}} d\left(Z_{1}^{-1}(d y+B)+Z_{1}^{-1}(d t-A)\right) .
\end{aligned}
$$

Note that there is no obvious easy split to be made by defining $\lambda_{1}=Z_{1}^{-1}-1$ and splitting off terms proportional to $\lambda_{1}$. This is because the fibres $A, B$ typically have singularities on the string profile and/or in the origin. So we can leave the well-behaved one-form $\Lambda$ implicit:

$$
2 H \equiv i_{K} G-d \Lambda=d\left(Z_{1}^{-1}(d v+\beta)\right)-d \Lambda,
$$

since the integrals we will perform are independent of $\Lambda$ anyway. In the explicit example of the once-wound circular profile, we can easily see that

$$
\frac{1}{L_{v}} \int_{\mathbb{R}^{2}} H=\frac{1}{L_{v}}\left(\frac{L_{v}}{2}\right)=\frac{1}{2},
$$

where we integrate the $\mathbb{R}^{2}$ cycle from the string profile (at $r=0, \theta=\pi / 2$ ) to $r=\infty$, and we used that $Z_{1}^{-1}(f=0)=0$ and $Z_{1}^{-1}(r=\infty)=1$.

We see that $H$ is the cohomological dual of the non-trivial two-cycle in the geometry, as expected. The harmonic part of the three-form $G$ and its dual $\tilde{G}$ are both proportional to the volume form of the non-trivial three-cycle $S^{3}$ :

$$
\frac{1}{2 \pi^{2}} \int_{S^{3}(\infty)} G=Q_{5}, \quad \frac{1}{2 \pi^{2}} \int_{S^{3}(\infty)} \tilde{G}=Q_{1},
$$

as these parts precisely define the D1 and D5 charges of the geometry. Putting this together gives for the null charge:

$$
\begin{aligned}
\mathcal{Q}_{K} & =-\frac{1}{4 \pi G_{6}} \int_{\mathbb{R}^{2} \times S^{3}}(H \wedge \tilde{G}+\tilde{H} \wedge G) \\
& =-\frac{1}{4 \pi G_{6}}\left(\int_{\mathbb{R}^{2}} H\right)(+1)\left(\int_{S^{3}} \tilde{G}\right)-\frac{1}{4 \pi G_{6}}\left(\int_{\mathbb{R}^{2}} \tilde{H}\right)(+1)\left(\int_{S^{3}} G\right) \\
& =-\frac{L_{v} \pi}{4 G_{6}}\left(Q_{1}+Q_{5}\right),
\end{aligned}
$$


where we used the intersection number to split the integral into separate integrals over the non-trivial cycles.

\subsection{D1-D5-P superstrata}

The most general three-charge microstate geometries that fall within six-dimensional supergravity arise from reduction on a rigid $T^{4}[22]$. These solutions excite all IIB supergravity fields in ten dimensions (metric, Ramond-Ramond fields $C_{(0)}, C_{(2)}, C_{(4)}$, as well as $B_{(2)}$ and the dilaton $\phi_{1}$ ). The solutions can be interpreted as solutions in minimal supergravity in six dimensions coupled to two tensor multiplets, see appendix B.

These solutions require extending the results of section 3.3 in two ways: considering an extra tensor multiplet, and adding the momentum charge P. Only then can we cover both generic D1-D5 geometries with a rigid $T^{4}[19]$ and the D1-D5-P superstrata [31]. However, these more general solutions are topologically equivalent to the D1-D5 supertubes (3.20). We will show that the Komar integral is unchanged.

The general superstrata solutions as given in $[25,31]$, in six-dimensional language, fit within the ansatz $[22,31]$ :

$$
\begin{aligned}
d s^{2} & =\frac{\mathcal{P}}{Z_{1} Z_{2}}\left(-\frac{2}{\sqrt{\mathcal{P}}}(d v+\beta)\left[d u+\omega+\frac{\mathcal{F}}{2}(d v+\beta)\right]+\sqrt{\mathcal{P}} d s_{4}^{2}\right), \\
e^{2 \phi} & =\frac{Z_{1}^{2}}{\mathcal{P}} \\
\chi & =\frac{Z_{4}}{Z_{1}} \\
2 B & =-\frac{Z_{2}}{\mathcal{P}}(d u+\omega) \wedge(d v+\beta)+a_{1} \wedge(d v+\beta)+\gamma_{2}, \\
B^{\prime} & =-\frac{Z_{4}}{\mathcal{P}}(d u+\omega) \wedge(d v+\beta)+a_{4} \wedge(d v+\beta)+\delta_{2}, \\
\mathcal{P} & =Z_{1} Z_{2}-Z_{4}^{2},
\end{aligned}
$$

where, similar to the D1-D5 ansatz, $d s_{4}^{2}$ is the $4 \mathrm{D}$ flat metric and $\beta, \omega, a_{1}, a_{4}, \gamma_{2}, \delta_{2}$ are forms on this $4 \mathrm{D}$ base. We refer to $[22,31]$ for the full set of supersymmetry equations and equations of motion and only quote those that we need:

$$
d \gamma_{2}=\star_{4} d Z_{2}, \quad d \delta_{2}=\star_{4} d Z_{4}
$$

The tensor $B$ comes from the dimensional reduction of $C_{(2)}$ while $B^{\prime}$ descends from $B_{(2)}$ in $10 \mathrm{D}$; the scalar $\phi$ is simply the $10 \mathrm{D}$ dilaton while $\chi$ is the $10 \mathrm{D}$ axion $C_{(0)}$. For more information on the dimensional reduction from $10 \mathrm{D}$ to $6 \mathrm{D}$ and the realization of the $\mathrm{SO}(1,2)$ symmetry, see appendix B. This ansatz reduces to the D1-D5 ansatz (3.20) when $Z_{4}=a_{4}=\delta_{2}=0$; the tensor multiplet parametrized by the fields $B^{\prime}, \chi$ is set to zero, truncating the $\mathrm{SO}(1,2)$ theory down to $\mathrm{SO}(1,1)$.

The tensor multiplet scalars $\tau=\chi+i e^{-\phi}$ parametrize the coset $\mathrm{SO}(1,2) / \mathrm{SO}(2)$. While $B$ and its field strength $G=d B$ are unconstrained, the tensor $B^{\prime}$ satisfies a duality relation. Indeed, the field strength:

$$
G^{\prime}=d B^{\prime}-2 \frac{\chi}{e^{-2 \phi}+\chi^{2}} d B
$$


is anti self-dual in six dimensions:

$$
G^{\prime}=-\star G^{\prime}
$$

Thus, we find the correct tensor field content for the $\mathrm{SO}(1,2)$ theory of minimal supergravity with two tensor multiplets.

The null charge is given by (see also appendix B):

$$
\mathcal{Q}_{K}=-\frac{1}{4 \pi G_{6}} \int_{V}(H \wedge \tilde{G}+\tilde{H} \wedge G)+\frac{1}{8 \pi G_{6}} \int_{V}\left(H^{\prime} \wedge G^{\prime}\right)
$$

where $H, \tilde{H}$ are defined as in (2.30), similarly $H^{\prime}$ is the harmonic part of $i_{K} G^{\prime}$, and the dual form $\tilde{G}$ is now defined by:

$$
\tilde{G}=\frac{e^{2 \phi}}{1+e^{2 \phi} \chi^{2}} \star G
$$

For the superstrata of [31], the terms in (3.49) involving $G, \tilde{G}$ can easily be seen to give the same contribution $\sim\left(Q_{1}+Q_{5}\right)$ as for the D1-D5 microstates above. The term involving $G^{\prime}$ does not contribute. It is easiest to realize this by seeing that $d B^{\prime}$ and $\chi d B$ fall off too fast at infinity to have a non-zero integral $\int_{S_{\infty}^{3}} G^{\prime}$; in essence, this is because $Z_{4}$ falls off faster at infinity than $Z_{1}$ or $Z_{2}$ (which give the $Q_{1}, Q_{5}$ contributions to the null charge as in the D1-D5 case above). ${ }^{8}$ We conclude that:

$$
\mathcal{Q}_{K}=-\frac{L_{v} \pi}{4 G_{6}}\left(Q_{1}+Q_{5}\right)
$$

just as for the D1-D5 supertube.

${ }^{8}$ To see this fall-off explicitly we quote the behaviour for the most general D1-D5 supertube invariant under $T^{4}$ rotations. This has five profile components $g_{i}, i=1 \ldots 4$ and $g_{5}$, and the fields are [45]:

$$
\begin{aligned}
& Z_{2}=1+\frac{Q_{5}}{L} \int_{0}^{L} \frac{1}{\left|x_{i}-g_{i}\left(v^{\prime}\right)\right|^{2}} d v^{\prime}, \quad Z_{4}=-\frac{Q_{5}}{L} \int_{0}^{L} \frac{\dot{g}_{5}\left(v^{\prime}\right)}{\left|x_{i}-g_{i}\left(v^{\prime}\right)\right|^{2}} d v^{\prime}, \\
& Z_{1}=1+\frac{Q_{5}}{L} \int_{0}^{L} \frac{\left|\dot{g}_{i}\left(v^{\prime}\right)\right|^{2}+\left|\dot{g}_{5}\left(v^{\prime}\right)\right|^{2}}{\left|x_{i}-g_{i}\left(v^{\prime}\right)\right|^{2}} d v^{\prime} \quad d \gamma_{2}=*_{4} d Z_{2} \quad d \delta_{2}=*_{4} d Z_{4}, \\
& A=-\frac{Q_{5}}{L} \int_{0}^{L} \frac{\dot{g}_{j}\left(v^{\prime}\right) d x^{j}}{\left|x_{i}-g_{i}\left(v^{\prime}\right)\right|^{2}} d v^{\prime} \\
& \beta=\frac{-A+B}{\sqrt{2}} \\
& \mathcal{F}=0, \\
& d B=-*_{4} d A \\
& \omega=\frac{-A-B}{\sqrt{2}} \\
& a_{1}=a_{4}=x_{3}=0 \text {. }
\end{aligned}
$$

An explicit example is a round profile in the $\mathbb{R}^{4}$ base and a non-zero $g_{5}$ component:

$$
g_{1}(v)=a \cos \left(v / R_{y}\right), \quad g_{2}(v)=a \sin \left(v / R_{y}\right), \quad g_{3}(v)=g_{4}(v)=0, \quad g_{5}(v)=-\frac{b}{k} \sin \left(v / R_{y}\right) .
$$

The D1-D5 seed solution of [31] starts from such a profile. Then we have that

$$
Z_{1}=1+\frac{Q_{1}}{f}+c_{1} \frac{\sin ^{2 k} \theta \cos (2 k \phi)}{\left(r^{2}+a^{2}\right)^{k} f}, \quad Z_{2}=1+\frac{Q_{5}}{f}, \quad Z_{4}=c_{4} \frac{\sin ^{k} \theta \cos (k \phi)}{\sqrt{r^{2}+a^{2}} f},
$$

where $c_{1}=\frac{Q_{1} a^{2} b^{2}}{2 a^{2}+b^{2}}$ and $c_{4}=\sqrt{\frac{Q_{1} Q_{5}}{a+2+b^{2} / 2}} b a^{k}$ are constants. Clearly $Z_{4}$ falls off too fast for the $H^{\prime} \wedge G^{\prime}$-term to contribute to the Komar integral. For superstrata solutions, we refer to [25, 31]. 
That the null charge gives the same result for D1-D5-P superstrata as for the D1-D5 supertubes is not so surprising from a topological point of view. The important thing to note is that a generic superstratum solution has the same topology as the D1-D5 round supertube. Superstrata describe fluctuations on top of a topologically non-trivial $S^{3}$ (shape modes depending on two variables), just as generic two-charge supertubes describe onedimensional shape modes on the $S^{3}$. This is the same $S^{3}$ present for the round supertube discussed in section 3.3, and therefore supertubes and superstrata have a similar topological three-cycle.

\section{Non-extremal example}

We now discuss the JMaRT solutions of [36], which have an interpretation as microstate geometries of the five-dimensional overspinning three-charge black hole. In the IIB frame, these are smooth solitons, with a natural interpretation in six-dimensional supergravity after dimensional reduction on the compact $T^{4}$.

\subsection{Metric and gauge fields}

The solitons are obtained by demanding the metric ansatz appropriate for describing the non-extremal three-charge black hole to be smooth. Usually, the five-dimensional physical charges are quoted, which in this case are the ADM mass $M_{\mathrm{ADM}, 5 \mathrm{D}}$, the electric charges $Q_{1}, Q_{5}, Q_{p}$, and the two angular momenta $J_{\psi}, J_{\phi}:{ }^{9}$

$$
\begin{aligned}
M_{\mathrm{ADM}, 5 \mathrm{D}} & =\frac{L_{y} \pi}{4 G_{6}} \frac{m}{2} \sum_{I} \cosh 2 \delta_{I}, & J_{\psi} & =-\frac{L_{y} \pi}{4 G_{6}} m\left(a_{1} c_{1} c_{2} c_{3}-a_{2} s_{1} s_{2} s_{3}\right), \\
Q_{I} & =\frac{m}{2} \sinh 2 \delta_{I}, & J_{\phi} & =-\frac{L_{y} \pi}{4 G_{6}} m\left(a_{2} c_{1} c_{2} c_{3}-a_{1} s_{1} s_{2} s_{3}\right),
\end{aligned}
$$

given in terms of parameters $m, \delta_{1}, \delta_{5}, \delta_{p}, a_{1}, a_{2}$ and with the notation $s_{i}=\sinh \delta_{i}, c_{i}=$ $\cosh \delta_{i}$. The supersymmetric limit is $m, a_{1}, a_{2} \rightarrow 0, \delta_{i} \rightarrow \infty$ while keeping $Q_{I}, m / \sqrt{a_{i}}$ fixed. We note that the $6 \mathrm{D} \mathrm{ADM}$ mass (for the asymptotically $\mathbb{R}^{4,1} \times S^{1}$ spacetime) is actually:

$$
M_{\mathrm{ADM}, 6 \mathrm{D}}=\frac{L_{y} \pi}{4 G_{6}} \frac{m}{2}\left(\cosh 2 \delta_{1}+\cosh 2 \delta_{5}+2 \cosh 2 \delta_{p}\right)
$$

so the contribution due to the momentum charge (which is the charge from the graviphoton in reducing from $6 \mathrm{D}$ to $5 \mathrm{D}$ ) is different.

\footnotetext{
${ }^{9}$ Standard conventions in the literature are to take $G_{5}=\pi / 4$, which would render the prefactor $L_{y} \pi /\left(4 G_{6}\right)=1$. As in the rest of the paper, we instead choose to keep the explicit factors of $G_{6}$ in all of the relevant formulae. We also choose a normalization for the $Q_{I}$ that is the same as the rest of the paper, instead of the usual normalization which would include a factor of $L_{y} \pi /\left(4 G_{6}\right)$ in the definition of the $Q_{I}$ as well.
} 
We choose to write the metric and gauge fields in the notation of [46]. The metric, scalar and gauge field in $6 \mathrm{D}$ are (note that $B=-C_{2} / 2$, with $C_{2}$ the RR two-form of [36]):

$$
\begin{aligned}
d s_{6}^{2}= & \frac{1}{H_{p}\left(H_{1} H_{5}\right)^{1 / 2}}\left[-H_{m}(d t+k)^{2}\right. \\
& \left.\quad+H_{p}^{2}\left(\left(d y+B_{p}^{m}+\frac{c_{p}}{s_{p}} k\right)+\frac{c_{p}}{s_{p}}\left(H_{p}^{-1}-1\right)(d t+k)\right)^{2}\right]+\left(H_{1} H_{5}\right)^{1 / 2} d s_{4}^{2} \\
e^{2 \sqrt{2} X}= & \frac{H_{1}}{H_{5}}, \\
-2 B= & \frac{c_{1}}{s_{1}} d t \wedge d y-\frac{c_{1}}{s_{1}} H_{1}^{-1}(d t+k) \wedge d y-B_{1} \wedge d z-\frac{c_{1} c_{p}}{s_{1} s_{p}} H_{1}^{-1} d t \wedge d k \\
& \quad-\frac{s_{p}}{c_{p}} d t \wedge B_{1}-\frac{c_{1}}{s_{1}} H_{1}^{-1} d t \wedge B_{3}+m s_{5} c_{5} \frac{r^{2}+a_{2}^{2}+m s_{1}^{2}}{f H_{1}} \cos ^{2} \theta d \psi \wedge d \phi
\end{aligned}
$$

where the quantities used are defined by:

$$
\begin{aligned}
d s_{4}^{2}= & f\left(\frac{r^{2}}{g} d r^{2}+d \theta^{2}+\sin ^{2} \theta d \phi^{2}+\cos ^{2} \theta d \psi^{2}\right) \\
& +H_{m}^{-1}\left(a_{1} \cos ^{2} \theta d \psi+a_{2} \sin ^{2} \theta d \phi\right)^{2}-\left(a_{2} \cos ^{2} \theta d \psi+a_{1} \sin ^{2} \theta d \phi\right)^{2} \\
k= & \frac{m}{f}\left[-\frac{c_{1} c_{5} c_{p}}{H_{m}}\left(a_{1} \cos ^{2} \theta d \psi+a_{2} \sin ^{2} \theta d \phi\right)+s_{1} s_{5} s_{p}\left(a_{2} \cos ^{2} \theta d \psi+a_{1} \sin ^{2} \theta d \phi\right)\right] \\
B^{(i)}= & \frac{m}{f H_{m}} \frac{c_{1} c_{5} c_{p}}{s_{I} c_{I}}\left(a_{1} \cos ^{2} \theta d \psi+a_{2} \sin ^{2} \theta d \phi\right)
\end{aligned}
$$

Everything is built from the following functions:

$$
\begin{aligned}
H_{i} & =1+\frac{m s_{i}^{2}}{f}, & H_{m} & =1-\frac{m}{f}, \\
f & =r^{2}+a_{1} \sin ^{2} \theta+a_{2}^{2} \cos ^{2} \theta, & g & =\left(r^{2}+a_{1}^{2}\right)\left(r^{2}+a_{2}^{2}\right)-m r^{2}=\left(r^{2}-r_{+}^{2}\right)\left(r^{2}-r_{-}^{2}\right),
\end{aligned}
$$

The three-form is simply $G=d B$. The dual potential, $\tilde{G}=d \tilde{B}$ is then given by:

$$
\tilde{B}=B \text { with } s_{1} \leftrightarrow s_{5} ; c_{1} \leftrightarrow c_{5} ; H_{1} \leftrightarrow H_{5}
$$

\subsection{Constraints for smooth solutions}

Smooth JMaRT solutions are determined for fixed charges $Q_{1}, Q_{5}, Q_{p}$, by two integers $m, n$. One can extend these to include $\mathbb{Z}_{k}$ orbifolds with $k$ an integer. They have the following relations between their parameters:

$$
\begin{aligned}
& r_{+}^{2}=-a_{1} a_{2} \frac{s_{1} s_{5} s_{p}}{c_{1} c_{5} c_{p}}, \\
& M=a_{1}^{2}+a_{2}^{2}-a_{1} a_{2}\left[\frac{c_{1}^{2} c_{5}^{2} c_{p}^{2}+s_{1}^{2} s_{5}^{2} s_{p}^{2}}{s_{1} c_{1} s_{5} c_{5} s_{p} c_{p}}\right] .
\end{aligned}
$$


The constant $t$ slices have the topology of $\mathbb{R}^{2} \times S^{3} / \mathbb{Z}_{k}$. The non-contractible $S^{3}$ is spanned at the origin $r=r_{+}$by the coordinates $\theta, \tilde{\psi}, \tilde{\phi}$, with the identifications

$$
\tilde{\psi}=\psi-\frac{s_{p} c_{p}}{a_{2} c_{1} c_{5} c_{p}-a_{1} s_{1} s_{5} s_{p}} y, \quad \tilde{\phi}=\phi-\frac{s_{p} c_{p}}{a_{1} c_{1} c_{5} c_{p}-a_{2} s_{1} s_{5} s_{p}} y,
$$

The following quantization conditions ensure that the identification $y \rightarrow y+2 \pi R$ is a closed orbit:

$$
\frac{s_{p} c_{p}}{a_{2} c_{1} c_{5} c_{p}-a_{1} s_{1} s_{5} s_{p}} R=m, \quad \frac{s_{p} c_{p}}{a_{1} c_{1} c_{5} c_{p}-a_{2} s_{1} s_{5} s_{p}} R=n,
$$

for integers $m, n$.

The $\mathbb{R}^{2}$ factor has a smooth origin at $r=r_{+}$, where the $t=$ constant part of the metric has the form (up to irrelevant constant prefactors)

$$
\left.d s^{2}\right|_{d t=0}=d \rho^{2}+\frac{\rho^{2}}{R^{2}} d y^{2},
$$

with the identification $y \sim y+2 \pi R k$ and the radius given by

$$
R=\frac{M s_{1} c_{1}}{\sqrt{a_{1} a_{2}}} \frac{\sqrt{s_{1} c_{1} s_{5} c_{5} s_{p} c_{p}}}{c_{1}^{2} c_{5}^{2} c_{p}^{2}-s_{1}^{2} s_{5}^{2} s_{p}^{2}} .
$$

\subsection{Komar integral}

We want to study the Komar integral, which reduces for this topology to

$$
\mathcal{Q}_{K}=-\frac{1}{4 \pi G_{6}} \int_{V}(H \wedge \tilde{G}+\tilde{H} \wedge G)=-\frac{1}{4 \pi G_{6}}\left(\int_{\mathbb{R}^{2}} H \int_{S^{3}} \tilde{G}+\int_{\mathbb{R}^{2}} \tilde{H} \int_{S^{3}} G\right)
$$

The non-contractible $S^{3}$ is homologically equivalent to the one at infinity appearing in Gauss' law. Hence we can perform the $S^{3}$ integral at spatial infinity:

$$
\begin{aligned}
\frac{1}{4 \pi G_{6}} \int_{S^{3}(\infty)} G & =-\frac{1}{8 \pi G_{6}} \lim _{r \rightarrow \infty} \int d\left[m s_{5} c_{5} \frac{r^{2}+a_{2}^{2}+m s_{1}^{2}}{f H_{1}} \cos ^{2} \theta d \psi \wedge d \phi\right] \\
& =-\left.\frac{\pi}{4 G_{6}} \lim _{r \rightarrow \infty} m s_{5} c_{5} \frac{r^{2}+a_{2}^{2}+m s_{1}^{2}}{f H_{1}} \cos ^{2} \theta\right|_{\theta=0} ^{\theta=\pi / 2}=\frac{\pi}{4 G_{6}} Q_{5}
\end{aligned}
$$

To obtain the $H$-integral, we can in principle split the interior product of the Killing vector with the three-form as

$$
i_{K} G=d \Lambda+H .
$$

However, for our purposes we do not need to do this explicitly: the integral of $i_{K} G$ and of $H$ are identical, as the contribution of $d \Lambda$ for $\Lambda$ a well-defined one-form cancels anyway.

To make contact with the supersymmetric limit later, we consider the Killing vector

$$
K=\partial_{t}+\alpha \partial_{y}
$$

with $\alpha$ a constant. Then we find that locally

$$
\left.d \omega \equiv i_{K} G\right|_{t=\text { const. }}, \quad \omega=\frac{c_{1}}{s_{1}} H_{1}^{-1}\left(d y+\left(\frac{c_{p}}{s_{p}}-\alpha\right) k+B^{(p)}\right)-\frac{c_{1}}{s_{1}} d y+\left(\frac{s_{p}}{c_{p}}-\alpha\right) B^{(1)} .
$$


The one-form $\omega$ is zero at infinity and well-behaved at any finite distance, but note that it is not globally well-defined. The integral $\int_{\mathbb{R}^{2}} i_{K} G_{3}$ only receives a contribution from the origin $r=r_{+}$. A short computation shows that for constant $\tilde{\psi}, \tilde{\phi}$ :

$$
\left.B^{(i)}\right|_{r=r_{+}}=-\frac{s_{p} c_{p}}{s_{i} c_{i}} d y,\left.\quad k\right|_{r=r_{+}}=0 .
$$

and hence the first bracket in (4.22) does not contribute in the $\mathbb{R}^{2}$-integral. The other terms give:

$$
\int_{\mathbb{R}^{2}} H=\int_{\mathbb{R}^{2}} i_{K} G=-\left.L_{y} \omega_{y}\right|_{r=r_{+}}=L_{y}\left(\frac{c_{1}}{s_{1}}+\frac{s_{p}^{2}-\alpha s_{p} c_{p}}{s_{1} c_{1}}\right)=L_{y} \frac{M_{1}+M_{p}-\alpha Q_{p}}{Q_{1}},
$$

using the notation

$$
M_{i}=\frac{m}{2} \cosh \left(2 \delta_{i}\right),
$$

which gives the contribution to the $5 \mathrm{D}$ ADM mass in the $i$-channel (so that $M_{\mathrm{ADM}, 5 \mathrm{D}}=$ $\left.\left(L_{y} \pi\right) /\left(4 G_{6}\right) \sum_{i} M_{i}\right)$.

In the end, we find that (4.17) becomes

$$
\begin{aligned}
\mathcal{Q}_{K} & =-\frac{L_{y} \pi}{4 G_{6}}\left(\frac{M_{5}+M_{p}-\alpha Q_{p}}{Q_{5}} Q_{5}+\frac{M_{1}+M_{p}-\alpha Q_{p}}{Q_{1}} Q_{1}\right) \\
& =-\frac{L_{y} \pi}{4 G_{6}}\left(M_{1}+M_{5}+2\left(M_{p}-\alpha Q_{p}\right)\right) .
\end{aligned}
$$

For $\alpha=0$, we have $K=\partial_{t}$ and we retrieve the 6D ADM mass (4.3) for the Komar charge $\mathcal{Q}_{K}$. Note that each term of the second line contributes to the $M_{p}$-channel. Also, in a sense, the non-extremality resides only in the integral over $H$; the integrals over $S^{3}$ of $G_{3}, \tilde{G}_{3}$ contribute the charge. For $\alpha=1$, so that $K=\partial_{t}+\partial_{y}$, the Komar charge in the supersymmetric limit becomes the usual null charge $\mathcal{Q}_{K}=-\left(L_{y} \pi\right) /\left(4 G_{6}\right)\left(Q_{1}+Q_{5}\right)$.

\section{Discussion and outlook}

Fluxes on non-trivial topology can support stationary configurations. This is a feature much used in microstate geometries and explained in detail in [13] for five-dimensional smooth microstates. We have explored the six-dimensional guise of this mechanism for horizonless solutions. The three-form field strengths of six-dimensional supergravity and the 2-, 3-cohomology play a crucial role and give a non-trivial contribution to the Komar integral (2.25) and thus to the conserved charges.

Many other avenues remain unexplored. One interesting direction is to explore the Smarr formula and the role of topology for non-flat asymptotics. As we have seen, compact directions give brane-like interpretations to the Komar integrals in terms of energy and tension densities. It would be interesting to understand the extension to asymptotic Antide Sitter spaces. In string theory, spaces of the asymptotic form $A d S_{p} \times S^{q}$ are very common. For these geometries, one must take care to regulate the Komar integral and perform a suitable background subtraction for the infinite AdS background contribution 
and render the Komar integrals finite. However, it does not seem that this subtraction term would be expressible in terms of an interesting topological integral. In six dimensions, supersymmetric microstate solutions have $A d S_{3} \times S^{3}$ core regions, and one can reinterpret our results for these geometries in their own right. In fact, for the D1-D5 solutions of section 3.3 it is clear that the relevant (non-trivial) three-cycle will be the $S^{3}$ and the two-cycle will be the ( $t=$ const.) non-compact spatial two-cycle of $A d S_{3}$. In other words, besides the subtlety of background subtraction, the situation for these $A d S_{3}$ geometries will be entirely analogous to the solutions considered here. Perhaps more enlightening would be $A d S_{5} \times S^{5}$ asymptotics, the arena of smooth LLM geometries [47]. The topological contribution to the Smarr formula for 1/16 BPS solutions might also shed light on possible smooth geometries with the asymptotics of the Gutowski-Reall black hole [48, 49].

Perhaps a similar discussion of topology can give us insight into the cosmological horizon. A Smarr formula has been discussed in the past [50, 51], but there has not been a discussion within supergravity models, nor with focus on topology. We leave such investigations, for instance for the de Sitter-Schwarzschild black hole, to future work.

One of the original motivations of this work was to understand how to discriminate between supersymmetric and non-supersymmetric smooth solution with non-zero Hawking temperature. The best studied example of the latter are the JMaRT solutions, which are smooth in six dimensions and hence fit in our current study. ${ }^{10}$ They have an ergoregion, which gives rise to an instability [57] that has been connected to Hawking radiation [58, 59]. One might expect that the appearance of an ergoregion in non-extremal microstate geometries is crucial for their decay and the connection to non-extremal black holes. Then one might also expect that the ergoregion plays a role in the universal characterization of microstate geometries through the Komar integral, as topology-supported solitons. However, the ergosurface is not topological and hence does not play a special role in the Komar integral. Hence the appearance of an ergoregion in the gravitational back-reaction of the probe constructions $[11,12]$ remains an open question.

\section{Acknowledgments}

We would like to thank D. Anninos, I. Bah, I. Bena, J. de Boer, S. Giusto, B. Niehoff, D. Turton, A. Van Proeyen, E. Verlinde and N. Warner for enlightening discussions. The research of B.V. is supported by the European Commission through the Marie Curie IntraEuropean fellowship 328652-QM-sing and B.V. is deeply grateful for the enormous support of Evelien Dejonghe. P.dL. most gratefully acknowledges support of the ERC through the Advanced Grant EMERGRAV. This work is part of the research programme of the Foundation for Fundamental Research on Matter (FOM), which is part of the Netherlands Organisation for Scientific Research (NWO).

\footnotetext{
${ }^{10}$ It would be interesting to study the various known five-dimensional non-extremal constructions, such as those based on JMaRT [52-54] and Bolt-like [55, 56] solutions.
} 


\section{A Uplift of five-dimensional multi-center solutions}

\section{A.1 General reduction}

Reducing 6D minimal supergravity plus a tensor multiplet gives the STU model in 5D. The $6 \mathrm{D}$ metric $\hat{g}_{a b}$ decomposes into the 5D metric $g_{a b}$, a graviphoton $A_{a}^{1}$, and a scalar $\phi_{2}$. The three-form gives two gauge fields: $\hat{G}_{a b c} \sim\left(\star_{5} F^{2}\right)_{a b c}$ and $\hat{G}_{a b 6} \sim F_{a b}^{3}$. Finally, our 6D scalar gives a scalar in $5 \mathrm{D} \hat{X}=\phi_{1}$. We can then reparametrize the $5 \mathrm{D}$ scalars $\phi_{1}, \phi_{2}$ to get the usual three constrained scalars $X^{I}$ of the STU model.

We use hats to denote $6 \mathrm{D}$ quantities in this section; unhatted quantities, such as indices, are 5D. We start with the 6D Lagrangian:

$$
\sqrt{-\hat{g}} \mathcal{L}_{6}=\sqrt{-\hat{g}}\left[\hat{R}-2 \partial_{\hat{\mu}} X \partial^{\hat{\mu}} X-\frac{1}{3} e^{2 \sqrt{2} \hat{X}} \hat{G}_{\hat{\mu} \hat{\nu} \hat{\rho}} \hat{G}^{\hat{\mu} \hat{\nu} \hat{\rho}}\right] .
$$

We call the (spacelike) coordinate along which we reduce $y$. The reduction ansatz for the metric is:

$$
d \hat{s}^{2}=e^{\phi_{2} / \sqrt{6}} d s_{5}^{2}+e^{-3 \phi_{2} / \sqrt{6}}\left(d y+A_{a}^{1} d x^{a}\right)^{2},
$$

with inverse:

$$
(\partial \hat{s})^{2}=e^{-\phi_{2} / \sqrt{6}}\left(\partial s_{5}\right)^{2}-2 e^{-\phi_{2} / \sqrt{6}} A^{1 \mu} \partial_{\mu} \partial_{y}+\left(e^{3 \phi_{2} / \sqrt{6}}+e^{-\phi_{2} / \sqrt{6}}\left(A^{1}\right)^{2}\right) \partial_{y}^{2} .
$$

The Einstein-Hilbert Lagrangian then reduces to:

$$
\frac{1}{G_{6}} \sqrt{-\hat{g}} \hat{R}=\frac{1}{G_{5}} \sqrt{-g}\left[R-\frac{1}{2}\left(\partial \phi_{2}\right)^{2}-\frac{1}{4} e^{-4 \phi_{2} / \sqrt{6} \phi_{2}}\left(F^{1}\right)^{2}\right],
$$

where $G_{6}=L_{y} G_{5}$. Note that $\sqrt{-\hat{g}}=e^{\phi_{2} / \sqrt{6}} \sqrt{-g_{5}}$.

The kinetic term for the $6 \mathrm{D}$ scalar $\hat{X}$ gives the contribution:

$$
\frac{1}{G_{6}} \sqrt{-\hat{g}}\left[-2 \partial_{\hat{\mu}} X \partial^{\hat{\mu}} X\right]=\frac{1}{G_{5}} \sqrt{-g}\left[-2\left(\partial \phi_{1}\right)^{2}\right] .
$$

Finally, reducing the three-form can be done most easily using form notation. The reduction ansatz is:

$$
2 \hat{G}=e^{-2 \sqrt{2} \phi_{1}+2 \phi_{2} / \sqrt{6}} \star_{5} F^{3}+F^{2} \wedge\left(d y+A^{1}\right),
$$

which also implies:

$$
2 \hat{\star} \hat{G}=e^{2 \phi_{2} / \sqrt{6}} \star_{5} F^{2}+e^{-2 \sqrt{2} \phi_{1}} F^{3} \wedge\left(d y+A^{1}\right) .
$$

Then the reduction of the kinetic term is:

$$
\begin{aligned}
2 e^{2 \sqrt{2} X_{\star} \hat{G} \wedge \hat{G}=d y \wedge\left[\frac{1}{2} e^{-2 \sqrt{2} \phi_{1}+2 \phi_{2} / \sqrt{6}} F_{3} \wedge \star_{5} F_{3}\right.} \\
\left.+\frac{1}{2} e^{2 \sqrt{2} \phi_{1}+2 \phi_{2} / \sqrt{6}} \star_{5} F_{2} \wedge F_{2}+F^{3} \wedge F^{2} \wedge A^{1}\right] .
\end{aligned}
$$


Summarizing, the reduction gives us the 5D Lagrangian:

$$
\begin{aligned}
\sqrt{-g} \mathcal{L}_{5}=\sqrt{-g}[ & R-\frac{1}{2}\left(\partial \phi_{2}\right)^{2}-\frac{1}{4} e^{-4 \phi_{2} / \sqrt{6} \phi_{2}}\left(F^{1}\right)^{2}-2\left(\partial \phi_{1}\right)^{2} \\
& \left.-\frac{1}{4} e^{2 \sqrt{2} \phi_{1}+2 \phi_{2} / \sqrt{6}}\left(F^{2}\right)^{2}-\frac{1}{4} e^{-2 \sqrt{2} \phi_{1}+2 \phi_{2} / \sqrt{6}}\left(F^{3}\right)^{2}\right]-\frac{1}{4} \epsilon^{\mu \nu \rho \sigma \lambda} A_{\mu}^{1} F_{\nu \rho}^{2} F_{\sigma \lambda}^{3} .
\end{aligned}
$$

To bring this to the usual STU form, we can define:

$$
X_{1}=e^{2 \phi_{2} / \sqrt{6}}, \quad X_{2}=e^{-\phi_{2} / \sqrt{6}-\sqrt{2} \phi_{1}}, \quad X_{3}=e^{-\phi_{2} / \sqrt{6}+\sqrt{2} \phi_{1}},
$$

so that $X^{1} X^{2} X^{3}=1$, and the Lagrangian can be written as:

$$
\mathcal{L}_{5}=R-\frac{1}{4} \frac{1}{\left(X^{I}\right)^{2}}\left(F^{I}\right)^{2}-\frac{1}{2} \frac{\left(\partial X^{I}\right)^{2}}{\left(X^{I}\right)^{2}}-\frac{1}{4} e^{-1} \epsilon^{\mu \nu \rho \sigma \lambda} A_{\mu}^{1} F_{\nu \rho}^{2} F_{\sigma \lambda}^{3},
$$

with sum over $I=\{1,2,3\}$ implied. This is the usual form of the STU Lagrangian. We can also write this as:

$$
\mathcal{L}_{5}=R-\frac{1}{2} Q_{I J} F_{\mu \nu}^{I} F^{J \mu \nu}-Q_{I J} \partial_{\mu} X^{I} \partial^{\mu} X^{J}-\frac{1}{24} e^{-1} C_{I J K} \epsilon^{\mu \nu \rho \sigma \lambda} A_{\mu}^{I} F_{\nu \rho}^{J} F_{\sigma \lambda}^{K},
$$

where we have $C_{I J K}=\left|\epsilon_{I J K}\right|$ and:

$$
\begin{aligned}
\frac{1}{6} C_{I J K} X^{I} X^{J} X^{K} & =1 \\
Q_{I J} & :=\frac{9}{2} X_{I} X_{J}-\frac{1}{2} C_{I J K} X^{K}, \\
X_{I} & :=\frac{1}{6} C_{I J K} X^{J} X^{K}
\end{aligned}
$$

\section{A.2 Uplifting SUSY solutions}

The most general $6 \mathrm{D}$ supersymmetric metric can be written as [35, 40]:

$$
\begin{aligned}
d s_{6}^{2} & =-2 H^{-1}(d v+\beta)\left[d u+\omega+\frac{\mathcal{F}}{2}(d v+\beta)\right]+H d x_{4}^{2}, \\
& =-H^{-1} \mathcal{F}\left[d v+\beta+\mathcal{F}^{-1}(d u+\omega)\right]^{2}+H^{-1} \mathcal{F}^{-1}(d u+\omega)^{2}+H d x_{4}^{2} .
\end{aligned}
$$

The rewriting of the metric in the second line shows us that we can reduce along $v$ as long as it is a spacelike coordinate, i.e. $\mathcal{F}<0$ everywhere. The reduction gives us:

$$
\begin{aligned}
d s_{5}^{2} & =-H^{-4 / 3} \mathcal{F}^{-2 / 3}(d u+\omega)^{2}+H^{2 / 3}\left(-\mathcal{F}^{1 / 3}\right) d x_{4}^{2}, \\
e^{-3 \phi_{2} / \sqrt{6}} & =H^{-1}(-\mathcal{F}), \\
A^{1} & =\beta+\mathcal{F}^{-1}(d u+\omega) .
\end{aligned}
$$

We see that the $6 \mathrm{D}$ null coordinate $u$ becomes a timelike coordinate in $5 \mathrm{D}$ [35].

With the metric, gauge fields and scalars in 5D given by (3.5)-(3.7), we can then identify the appropriate $6 \mathrm{D}$ quantities in terms of the $5 \mathrm{D}$ ones as follows:

$$
\mathcal{F}=-Z_{1}, \quad \omega=k, \quad \beta=B^{1}, \quad H=\left(Z_{2} Z_{3}\right)^{1 / 2} .
$$


For reference, the full $6 \mathrm{D}$ fields are given by:

$$
\begin{aligned}
d s_{6}^{2}=-\frac{1}{Z_{1}\left(Z_{2} Z_{3}\right)^{1 / 2}}(d u+k)^{2}+\left(Z_{2} Z_{3}\right)^{1 / 2} d s_{4}^{2} \\
+\frac{Z_{1}}{\left(Z_{2} Z_{3}\right)^{1 / 2}}\left(d v-Z_{1}^{-1}(d u+k)+B^{1}\right)^{2}, \\
e^{\sqrt{2} X}=e^{\sqrt{2} \phi_{1}}=X_{1}^{1 / 2} X_{3}=\frac{Z_{2}^{1 / 2}}{Z_{3}^{1 / 2}} . \\
2 G=X_{3}^{-2} \star_{5} F^{3}+F^{2} \wedge\left(d v+A^{1}\right) .
\end{aligned}
$$

\section{B Rigid $T^{4}$ reduction of IIB and $\mathrm{SO}(1,2)$ truncation}

The reduction of IIB supergravity to six-dimensional $\mathcal{N}=(1,0)$ supergravity with 2 tensor multiplets goes in two steps. In a first step, reduction of the bosonic sector on a rigid $T^{4}$ gives a theory with $\mathrm{SO}(2,2)$ global symmetry [60]. Then the compatibility with D1-D5-P supersymmetries as in [22] leads to the bosonic sector of the $\mathrm{SO}(1,2)$ invariant supergravity.

First, we reduce IIB supergravity on a $T^{4}$, keeping only the components of the fields with indices over the remaining six dimensions. This gives us two dilatons (from the 10D dilaton $\phi$ and the breathing mode of the $T^{4}$ ); two axions (from the 10D axion $C_{(0)}$ and from the only relevant component of $C_{(4)}$ ), along with the two reduced three-forms coming from the potentials $C_{(2)}$ and $B_{(2)}$. The reduction ansatz is [60,61]:

$$
\begin{aligned}
d s_{10, s t r}^{2} & =e^{\phi_{1} / 2}\left(e^{\phi_{2} / 2} d s_{6}^{2}+e^{-\phi_{2} / 2} d s_{T_{4}}^{2}\right), & & C_{(0)}=\chi_{1}, \\
\phi & =\phi_{1}, & & C_{(2)}=C_{(2)}, \\
B_{(2)} & =B_{(2)}, & & C_{(4)}=-\chi_{2} \operatorname{vol}\left(T_{4}\right)+\cdots,
\end{aligned}
$$

where $d s_{T_{4}}^{2}$ and $\operatorname{vol}\left(T^{4}\right)$ are the flat metric and flat volume element on $T^{4}$. The $\cdots$ in $C_{(4)}$ are other terms that follow from the self-duality condition $F_{(5)}=\star F_{(5)}$. Note that we use the IIB supergravity conventions as in [31]. The resulting 6D Lagrangian is [60]:

$$
\begin{aligned}
\mathcal{L}_{6 D, \mathrm{SO}(2,2)}= & R-\frac{1}{2}\left(\partial \phi_{1}\right)^{2}-\frac{1}{2}\left(\partial \phi_{2}\right)^{2}-\frac{1}{2} e^{2 \phi_{1}}\left(\partial \chi_{1}\right)^{2}-\frac{1}{2} e^{2 \phi_{2}}\left(\partial \chi_{2}\right)^{2} \\
& -\frac{1}{12} e^{-\phi_{1}-\phi_{2}} H_{(3)}^{2}-e^{\phi_{1}-\phi_{2}} \frac{1}{12} F_{(3)}^{2}+\chi_{2} H_{(3)} \wedge d C_{(2)},
\end{aligned}
$$

with $F_{(3)} \equiv d C_{(2)}-C_{(0)} H_{(3)}$. This reduction/truncation has an $\mathrm{SO}(2,2) \cong \mathrm{SL}(2)_{1} \times \mathrm{SL}(2)_{2}$ symmetry where each $\tau_{i}=\chi_{i}+i e^{-\phi_{i}}$ parametrizes an $\mathrm{SL}(2) / \mathrm{SO}(2)$ coset. The $\mathrm{SO}(2,2)$ is not a symmetry of the tensor Lagrangian, but rather of the equations of motion and Bianchi identities. Those can be written as Bianchi identities of an $\mathrm{SO}(2,2)$ vector of field strengths $G^{r}$ with components

$$
\left(\begin{array}{c}
G^{1} \\
G^{2}
\end{array}\right) \equiv\left(\begin{array}{c}
d B_{(2)} \\
d C_{(2)}
\end{array}\right), \quad\left(\begin{array}{c}
G^{3} \\
G^{4}
\end{array}\right) \equiv\left(\begin{array}{c}
\frac{d \mathcal{L}_{6 D}}{d G^{2}} \\
-\frac{d \mathcal{L}_{6 D}}{d G^{1}}
\end{array}\right)=-e^{\phi_{2}}\left(i \sigma_{2}\right) \cdot \mathcal{M}_{1} \cdot\left(\begin{array}{c}
\star G^{1} \\
\star G^{2}
\end{array}\right)+\chi_{2}\left(\begin{array}{c}
G^{1} \\
G^{2}
\end{array}\right) .
$$


Those tensors obey the duality relation (compare (2.19)):

$$
\mathcal{M}_{r s} G^{s}=\eta_{r s} \star G^{s},
$$

with the off-diagonal $\mathrm{SO}(2,2)$ metric $\eta=\left(i \sigma_{2}\right) \otimes\left(i \sigma_{2}\right)$ and scalar matrix

$$
\mathcal{M}=\mathcal{M}_{2}\left(\tau_{2}\right) \otimes \mathcal{M}_{1}\left(\tau_{1}\right), \quad \text { with } \quad \mathcal{M}_{i}=V_{i} V_{i}^{T}, \quad V_{i}=\left(\begin{array}{cc}
e^{-\frac{1}{2} \phi_{i}} & \chi_{i} e^{\frac{1}{2} \phi_{i}} \\
0 & e^{\frac{1}{2} \phi_{i}}
\end{array}\right) .
$$

It is important to realize that this $\mathrm{SO}(2,2)$ theory cannot be the bosonic part of any supergravity theory. One can perform a further truncation to obtain a theory that can be the bosonic part of $\mathrm{SO}(1,2) \cong \mathrm{SL}(2)$ supergravity by setting $\tau_{2}=f\left(\tau_{1}\right)$ with $f$ an $\mathrm{SL}(2)$ transformation. This identifies a 'diagonal' $\mathrm{SL}(2)$ subgroup in $\mathrm{SO}(2,2) \cong \mathrm{SL}(2)_{1} \times \mathrm{SL}(2)_{2}$. The four tensors $G^{r}$ then decompose in a singlet and a triplet under this truncation. Consistency of the truncation requires that we put the singlet to zero.

We are interested in solutions with the supersymmetries of the D1-D5-P system [22], giving the truncation:

$$
\tau_{2}=-\frac{1}{\tau_{1}}
$$

The $\tau_{2}$ equation of motion then requires that we put the singlet $G^{1}+G^{4}$ to zero. The remaining three field strengths are

$$
\hat{G}^{1}=\frac{1}{2}\left(G^{3}-G^{2}\right), \quad \hat{G}^{2}=\frac{1}{2}\left(G^{2}+G^{3}\right), \quad \hat{G}^{3}=\frac{1}{2}\left(G^{4}-G^{1}\right),
$$

Dropping the hats again, $G^{r}$ then obeys the self-duality relation with the $\mathrm{SO}(1,2)$ matrix

$$
V=\exp \left(\chi E_{+}\right) \exp (\phi H / 2), \quad E_{+}=\left(\begin{array}{ccc}
0 & 0 & 1 \\
0 & 0 & 1 \\
1 & -1 & 0
\end{array}\right), \quad H=\left(\begin{array}{ccc}
0 & 2 & 0 \\
2 & 0 & 0 \\
0 & 0 & 0
\end{array}\right)
$$

and the Komar integral (2.25) applies.

To make the connection to the theory with one tensor multiplet clear, we write the vanishing singlet as an anti self-duality constraint on a three-form $G^{\prime}$ :

$$
G^{\prime}=-\star G^{\prime}, \quad G^{\prime}=d B_{(2)}+\chi_{2} d C_{(2)}=\frac{e^{-2 \phi_{1}} H_{(3)}-\chi_{1} F_{(3)}}{e^{-2 \phi_{1}}+\chi_{1}^{2}} .
$$

We can then take $G \equiv(1 / 2) d C_{(2)}$ to be the (unrestricted) tensor that is the combination of the other self-dual and anti self-dual tensors. In section 3.4, we take $\phi=\phi_{1}, \chi=\chi_{1}$ and $B=(1 / 2) C_{(2)}, B^{\prime}=B_{(2)}$. An obvious further truncation of this $\mathrm{SO}(1,2)$ theory is to take $G^{\prime}=0, \chi_{1}=0$ which leaves us with the $\mathrm{SO}(1,1)$ sector used in large parts of this paper, after the identification $\phi_{1}=\sqrt{2} X$.

For the $\mathrm{SO}(1,2)$ theory with the unrestricted three-form $G$ and the anti self-dual threeform $G^{\prime}$ as defined above, the generalization (2.24) of (2.29) for the null charge reduces to:

$$
\mathcal{Q}_{K}=-\frac{1}{4 \pi G_{6}} \int_{V}(H \wedge \tilde{G}+\tilde{H} \wedge G)+\frac{1}{8 \pi G_{6}} \int_{V}\left(H^{\prime} \wedge G^{\prime}\right)
$$


where $H, \tilde{H}$ are defined as in (2.30), keeping in mind the $\mathrm{SO}(1,2)$-generalized definitions for the dual form:

$$
\tilde{G}=\frac{e^{2 \phi_{1}}}{1+e^{2 \phi_{1}} \chi_{1}^{2}} \star G
$$

The harmonic form $H^{\prime}$ is defined by the split:

$$
i_{K} G^{\prime}=d \Lambda^{\prime}+H^{\prime}
$$

where $\Lambda^{\prime}$ is a globally defined one-form.

Open Access. This article is distributed under the terms of the Creative Commons Attribution License (CC-BY 4.0), which permits any use, distribution and reproduction in any medium, provided the original author(s) and source are credited.

\section{References}

[1] I. Bena and N.P. Warner, Black holes, black rings and their microstates, Lect. Notes Phys. 755 (2008) 1 [hep-th/0701216] [INSPIRE].

[2] S.D. Mathur, Fuzzballs and the information paradox: a summary and conjectures, arXiv:0810.4525 [INSPIRE].

[3] V. Balasubramanian, J. de Boer, S. El-Showk and I. Messamah, Black holes as effective geometries, Class. Quant. Grav. 25 (2008) 214004 [arXiv:0811.0263] [INSPIRE].

[4] K. Skenderis and M. Taylor, The fuzzball proposal for black holes, Phys. Rept. 467 (2008) 117 [arXiv:0804.0552] [INSPIRE].

[5] B.D. Chowdhury and A. Virmani, Modave lectures on fuzzballs and emission from the D1-D5 system, arXiv:1001.1444.

[6] I. Bena and N.P. Warner, Resolving the structure of black holes: philosophizing with a hammer, arXiv:1311.4538 [INSPIRE].

[7] S.L. Braunstein, S. Pirandola and K. Życzkowski, Better late than never: information retrieval from black holes, Phys. Rev. Lett. 110 (2013) 101301 [arXiv:0907.1190] [INSPIRE].

[8] S.D. Mathur, The information paradox: a pedagogical introduction, Class. Quant. Grav. 26 (2009) 224001 [arXiv:0909.1038] [INSPIRE].

[9] A. Almheiri, D. Marolf, J. Polchinski and J. Sully, Black holes: complementarity or firewalls?, JHEP 02 (2013) 062 [arXiv:1207.3123] [INSPIRE].

[10] P. Breitenlohner, D. Maison and G.W. Gibbons, Four-dimensional black holes from Kaluza-Klein theories, Commun. Math. Phys. 120 (1988) 295.

[11] I. Bena, A. Puhm and B. Vercnocke, Metastable supertubes and non-extremal black hole microstates, JHEP 04 (2012) 100 [arXiv:1109.5180] [INSPIRE].

[12] I. Bena, A. Puhm and B. Vercnocke, Non-extremal black hole microstates: fuzzballs of fire or fuzzballs of fuzz?, JHEP 12 (2012) 014 [arXiv:1208.3468] [INSPIRE].

[13] G.W. Gibbons and N.P. Warner, Global structure of five-dimensional fuzzballs, Class. Quant. Grav. 31 (2014) 025016 [arXiv:1305.0957] [INSPIRE].

[14] P.A. Haas, Smarr's formula in eleven-dimensional supergravity, arXiv:1405.3708 [INSPIRE]. 
[15] G. Compere, K. Copsey, S. de Buyl and R.B. Mann, Solitons in five dimensional minimal supergravity: local charge, exotic ergoregions and violations of the BPS bound, JHEP 12 (2009) 047 [arXiv:0909.3289] [INSPIRE].

[16] O. Lunin and S.D. Mathur, Metric of the multiply wound rotating string, Nucl. Phys. B 610 (2001) 49 [hep-th/0105136] [INSPIRE].

[17] O. Lunin and S.D. Mathur, AdS/CFT duality and the black hole information paradox, Nucl. Phys. B 623 (2002) 342 [hep-th/0109154] [INSPIRE].

[18] O. Lunin, J.M. Maldacena and L. Maoz, Gravity solutions for the D1-D5 system with angular momentum, hep-th/0212210 [INSPIRE].

[19] I. Kanitscheider, K. Skenderis and M. Taylor, Fuzzballs with internal excitations, JHEP 06 (2007) 056 [arXiv:0704.0690] [INSPIRE].

[20] I. Bena, J. de Boer, M. Shigemori and N.P. Warner, Double, double supertube bubble, JHEP 10 (2011) 116 [arXiv:1107.2650] [InSPIRE].

[21] I. Bena, M. Shigemori and N.P. Warner, Black-hole entropy from supergravity superstrata states, JHEP 10 (2014) 140 [arXiv:1406.4506] [INSPIRE].

[22] S. Giusto, L. Martucci, M. Petrini and R. Russo, 6D microstate geometries from $10 D$ structures, Nucl. Phys. B 876 (2013) 509 [arXiv:1306.1745] [INSPIRE].

[23] I. Kanitscheider, K. Skenderis and M. Taylor, Holographic anatomy of fuzzballs, JHEP 04 (2007) 023 [hep-th/0611171] [INSPIRE].

[24] S. Giusto, R. Russo and D. Turton, New D1-D5-P geometries from string amplitudes, JHEP 11 (2011) 062 [arXiv:1108.6331] [INSPIRE].

[25] S. Giusto and R. Russo, Perturbative superstrata, Nucl. Phys. B 869 (2013) 164 [arXiv:1211.1957] [INSPIRE].

[26] O. Lunin, Adding momentum to D1-D5 system, JHEP 04 (2004) 054 [hep-th/0404006] [INSPIRE].

[27] S. Giusto, S.D. Mathur and A. Saxena, Dual geometries for a set of 3-charge microstates, Nucl. Phys. B 701 (2004) 357 [hep-th/0405017] [INSPIRE].

[28] S. Giusto, S.D. Mathur and A. Saxena, 3-charge geometries and their CFT duals, Nucl. Phys. B 710 (2005) 425 [hep-th/0406103] [INSPIRE].

[29] I. Bena, S.F. Ross and N.P. Warner, On the oscillation of species, JHEP 09 (2014) 113 [arXiv: 1312.3635] [INSPIRE].

[30] I. Bena, S.F. Ross and N.P. Warner, Coiffured black rings, Class. Quant. Grav. 31 (2014) 165015 [arXiv:1405.5217] [INSPIRE].

[31] I. Bena et al., Habemus superstratum! a constructive proof of the existence of superstrata, arXiv: 1503.0146.

[32] S. Deser and M. Soldate, Gravitational energy in spaces with compactified dimensions, Nucl. Phys. B 311 (1989) 739 [INSPIRE].

[33] K. Stelle, BPS branes in supergravity, hep-th/9803116.

[34] P.K. Townsend and M. Zamaklar, The first law of black brane mechanics, Class. Quant. Grav. 18 (2001) 5269 [hep-th/0107228] [INSPIRE]. 
[35] J.B. Gutowski, D. Martelli and H.S. Reall, All supersymmetric solutions of minimal supergravity in six-dimensions, Class. Quant. Grav. 20 (2003) 5049 [hep-th/0306235] [INSPIRE].

[36] V. Jejjala, O. Madden, S.F. Ross and G. Titchener, Non-supersymmetric smooth geometries and D1-D5-P bound states, Phys. Rev. D 71 (2005) 124030 [hep-th/0504181] [INSPIRE].

[37] A.W. Peet, TASI lectures on black holes in string theory, hep-th/0008241.

[38] S. Ferrara, F. Riccioni and A. Sagnotti, Tensor and vector multiplets in six-dimensional supergravity, Nucl. Phys. B 519 (1998) 115 [hep-th/9711059] [INSPIRE].

[39] F. Riccioni, All couplings of minimal six-dimensional supergravity, Nucl. Phys. B 605 (2001) 245 [hep-th/0101074] [INSPIRE].

[40] M. Cariglia and O.A.P. Mac Conamhna, The General form of supersymmetric solutions of $N=(1,0) \mathrm{U}(1)$ and $\mathrm{SU}(2)$ gauged supergravities in six-dimensions, Class. Quant. Grav. 21 (2004) 3171 [hep-th/0402055] [INSPIRE].

[41] I. Bena, S. Giusto, M. Shigemori and N.P. Warner, Supersymmetric solutions in six dimensions: a linear structure, JHEP 03 (2012) 084 [arXiv:1110.2781] [INSPIRE].

[42] J.P. Gauntlett and J.B. Gutowski, General concentric black rings, Phys. Rev. D 71 (2005) 045002 [hep-th/0408122] [INSPIRE].

[43] H. Elvang, R. Emparan, D. Mateos and H.S. Reall, Supersymmetric black rings and three-charge supertubes, Phys. Rev. D 71 (2005) 024033 [hep-th/0408120] [INSPIRE].

[44] I. Bena and N.P. Warner, One ring to rule them all... and in the darkness bind them?, Adv. Theor. Math. Phys. 9 (2005) 667 [hep-th/0408106] [INSPIRE].

[45] S. Giusto and R. Russo, Superdescendants of the D1-D5 CFT and their dual 3-charge geometries, JHEP 03 (2014) 007 [arXiv:1311.5536] [INSPIRE].

[46] B.D. Chowdhury and D.R. Mayerson, Multi-centered D1-D5 solutions at finite B-moduli, JHEP 02 (2014) 043 [arXiv: 1305.0831] [INSPIRE].

[47] H. Lin, O. Lunin and J.M. Maldacena, Bubbling AdS space and 1/2 BPS geometries, JHEP 10 (2004) 025 [hep-th/0409174] [INSPIRE].

[48] J.B. Gutowski and H.S. Reall, Supersymmetric AdS 5 black holes, JHEP 02 (2004) 006 [hep-th/0401042] [INSPIRE].

[49] J.B. Gutowski and H.S. Reall, General supersymmetric $A d S_{5}$ black holes, JHEP 04 (2004) 048 [hep-th/0401129] [INSPIRE].

[50] G.W. Gibbons and S.W. Hawking, Cosmological event horizons, thermodynamics and particle creation, Phys. Rev. D 15 (1977) 2738 [InSPIRE].

[51] L.F. Abbott and S. Deser, Charge definition in nonabelian gauge theories, Phys. Lett. B 116 (1982) 259 [inSPIRE].

[52] G. Bossard and S. Katmadas, Floating JMaRT, JHEP 04 (2015) 067 [arXiv: 1412.5217] [INSPIRE].

[53] D. Katsimpouri, A. Kleinschmidt and A. Virmani, An inverse scattering construction of the JMaRT fuzzball, JHEP 12 (2014) 070 [arXiv:1409.6471] [INSPIRE].

[54] S. Banerjee, B.D. Chowdhury, B. Vercnocke and A. Virmani, Non-supersymmetric microstates of the MSW system, JHEP 05 (2014) 011 [arXiv:1402.4212] [INSPIRE]. 
[55] I. Bena, S. Giusto, C. Ruef and N.P. Warner, A (running) bolt for new reasons, JHEP 11 (2009) 089 [arXiv:0909.2559] [INSPIRE].

[56] G. Bossard and S. Katmadas, A bubbling bolt, JHEP 07 (2014) 118 [arXiv: 1405.4325] [INSPIRE].

[57] V. Cardoso, O.J.C. Dias and R.C. Myers, On the gravitational stability of D1-D5-P black holes, Phys. Rev. D 76 (2007) 105015 [arXiv:0707.3406] [INSPIRE].

[58] B.D. Chowdhury and S.D. Mathur, Radiation from the non-extremal fuzzball, Class. Quant. Grav. 25 (2008) 135005 [arXiv:0711.4817] [InSPIRE].

[59] B.D. Chowdhury and S.D. Mathur, Non-extremal fuzzballs and ergoregion emission, Class. Quant. Grav. 26 (2009) 035006 [arXiv:0810.2951] [INSPIRE].

[60] M.J. Duff, H. Lü and C.N. Pope, $A d S_{3} \times S^{3}$ (un)twisted and squashed and an $O(2,2, Z)$ multiplet of dyonic strings, Nucl. Phys. B 544 (1999) 145 [hep-th/9807173] [INSPIRE].

[61] I.V. Lavrinenko, H. Lü, C.N. Pope and T.A. Tran, U duality as general coordinate transformations and space-time geometry, Int. J. Mod. Phys. A 14 (1999) 4915 [hep-th/9807006] [INSPIRE]. 